\title{
Deterministic Simulation of Random Processes
}

\author{
By Joel N. Franklin
}

1. Introduction. For many problems in engineering, economics, mathematics, and the sciences we are required to simulate random processes. The simulation is usually effected by a computer program which generates a non-random, deterministic sequence of numbers $x_{1}, x_{2}, \cdots$ which is supposed to resemble a sequence of independent, random samples from the uniform probability distribution on the interval $0 \leqq x<1$. The purpose of this paper is to define some general properties of random sequences and to investigate certain deterministic sequences which have some or all of these properties. We shall ignore the limitation that a digital computer with a finite word-length and a finite memory, operating under a single stored program, can produce only sequences of limited precision which are ultimately periodic. This limitation is a kind of round-off error. We shall take as a model of a deterministic mechanism any of the stored-program digital computers now commonly used for scientific computation modified in a single respect: let the wordlength be infinite; let rational and irrational numbers $x$ be recorded and computed with perfect precision.

The fundamental problem approached in this paper is to construct an infinite, deterministic sequence $x_{n}$ which has every property shared by all infinite, random sequences of independent samples from the uniform distribution.

Equidistribution is a first requirement of randomness. The sequence $x_{n}$ is equidistributed in $0 \leqq x<1$ if, for $0 \leqq a<b \leqq 1$,

$$
\lim _{N \rightarrow \infty} \frac{1}{N} \sum_{\substack{a \leqq x_{n}<b \\ 1 \leqq n \leqq N}} 1=b-a .
$$

H. Weyl [1] showed that the fractional parts $x_{n}=\{n \alpha\}$ are equidistributed for any irrational $\alpha$. A summary of results on equidistribution is given by J. F. Koksma [4]. A sequence in $r$ dimensions $z^{(n)}=\left(z_{1}{ }^{(n)}, z_{2}{ }^{(n)}, \cdots, z_{r}{ }^{(n)}\right)$ is equidistributed in the unit cube

$$
C_{r}: 0 \leqq z_{1}<1, \quad 0 \leqq z_{2}<1, \quad \cdots, \quad 0 \leqq z_{r}<1
$$

if, for $0 \leqq a_{i}<b_{i} \leqq 1 \quad(i=1, \cdots, r)$,

$$
\lim _{N \rightarrow \infty} \frac{1}{N} \sum_{\substack{a_{i} \leqq z_{i}(n)<b_{i}(i=1, \cdots, r) \\ 1 \leqq n \leqq N}} 1=\prod_{i=1}^{r}\left(b_{i}-a_{i}\right) .
$$

It was shown by Weyl [1] and by van der Corput [2] that the sequence $z^{(n)}$ is equidistributed if and only if

$$
\lim _{N \rightarrow \infty} \frac{1}{N} \sum_{n=1}^{N} \exp 2 \pi i\left(k_{1} z_{1}^{(n)}+k_{2} z_{2}^{(n)}+\cdots+k_{r}{z_{r}}^{(n)}\right)=0
$$

for every set of integers $k_{1}, \cdots, k_{r}$ not all zero.

Received January 16, 1962. 
We require a definition of "probability" for deterministic sequences $x_{n}$. Let $S_{n}$ be a sequence of statements about the numbers $x_{n}$. We define

$$
\operatorname{Pr}\left(S_{n}\right)=\lim _{N \rightarrow \infty} \frac{1}{N} \sum_{\substack{S_{n} \text { true } \\ 1 \leqq n \leqq N}} 1
$$

when this limit exists. For example, we define

$$
\operatorname{Pr}\left(x_{n}>x_{n+1}\right)=\lim _{N \rightarrow \infty} \frac{1}{N} \sum_{\substack{x_{n}>x_{n}+1 \\ 1 \leqq n \leqq N}} 1
$$

if the limit exists. As another example, definition (1.1) states that a sequence $x_{n}$ is equidistributed in $C_{1}$ if $\operatorname{Pr}\left(a \leqq x_{n}<b\right)=b-a$ for $0 \leqq a<b \leqq 1$.

There are $k$ ! possible orderings of $k$ distinct numbers $z_{1}, z_{2}, \cdots, z_{k}$. Correspondingly, there are $k$ ! classes $O_{j}(j=1, \cdots, k !)$ of vectors $\left(z_{1}, \cdots, z_{k}\right)$. For example, if $k=2$ we say $\left(z_{1}, z_{2}\right) \in O_{1}$ if $z_{1}>z_{2}$, but $\left(z_{1}, z_{2}\right) \in O_{2}$ if $z_{2}>z_{1}$. For a given sequence $x_{n}$ in $C_{1}$ define the $k$-dimensional vectors

$$
z^{(n)}=\left(x_{n}, x_{n+1}, \cdots, x_{n+k-1}\right) \quad(n=1,2, \cdots) .
$$

We shall say that the sequence $x_{n}$ is equipartitioned by $k$ 's if

$$
\operatorname{Pr}\left(z^{(n)} \in O_{j}\right)=\frac{1}{k !} \quad(j=1, \cdots, k !) .
$$

The sequence $x_{n}$ will be called equidistributed by $k$ 's if the $k$-dimensional sequence $z^{(n)}$ is equidistributed in the unit cube $C_{k}$. The sequence $x_{n}$ is completely equidistributed if it is equidistributed by $k$ 's for every $k$.

More generally, the sequence of $r$-dimensional vectors

$$
y^{(n)}=\left(y_{1}^{(n)}, \cdots, y_{r}^{(n)}\right) \quad(n=1,2, \cdots)
$$

is defined to be equidistributed by $k$ 's if the sequence of $k \cdot r$ dimensional vectors

$$
w^{(n)}=\left(y_{1}{ }^{(n)}, \cdots, y_{r}^{(n)}, y_{1}^{(n+1)}, \cdots, y_{r}^{(n+1)}, \cdots, y_{1}{ }^{(n+k-1)}, \cdots, y_{r}^{(n+k-1)}\right)
$$

is equidistributed in $C_{k r}$. The sequence $y^{(n)}$ is completely equidistributed if it is equidistributed by $k$ 's for every $k$.

For one-dimensional equidistributed sequences $x_{n}$ we define the autocorrelation function $R(\tau)$ and the spectral density $\phi(\omega)$ by

$$
\begin{aligned}
& R(\tau)=\lim _{N \rightarrow \infty} \frac{1}{N} \sum_{n=1}^{N}\left(x_{n}-\frac{1}{2}\right)\left(x_{n+\tau}-\frac{1}{2}\right) \quad(\tau=0,1, \cdots) \\
& \phi(\omega)=R(0) \omega+2 \sum_{\tau=1}^{\infty} R(\tau) \cos 2 \pi \tau \omega
\end{aligned}
$$

if these limits exist. D. L. Jagerman [5] has proved that, if the limit

$$
F(\tau, k, \nu)=\lim _{N \rightarrow \infty} \frac{1}{N} \sum_{j=1}^{N} \sin 2 \pi k x_{j} \sin \nu x_{j+r}
$$

exists, then the equidistributed sequence $x_{n}$ has the autocorrelation function

$$
R(\tau)=\sum_{k=1}^{\infty} \sum_{\nu=1}^{\infty} \frac{F(\tau, k, \nu)}{\pi^{2} k \nu} .
$$


The numbers $x_{n}$ form a white sequence if $R(\tau)=0$ for all $\tau \neq 0$. Using the theorem (1.11) Jagerman proved that $x_{n}=\left\{n^{2} \alpha\right\}$ is white.

Finally, for the purpose of generating multi-dimensional sequences from onedimensional sequences, we define the $r$-dimensional derived sequence $y^{(n)}$ related to the one-dimensional sequence $x_{n}$ by the formula

$$
y^{(n)}=\left(x_{n r}, x_{n r+1}, \cdots, x_{n r+r-1}\right) \quad(n=1,2, \cdots) .
$$

All of the properties equipartition, equidistribution, and whiteness are of interest because they are properties of the truly random sequences which we are trying to simulate. In this paper these properties are studied with regard to several classes of equidistributed sequences. We consider briefly the Weyl sequence $x_{n}=$ $\{n \alpha\}, \alpha$ irrational. We then consider Multiply sequences, which are formed from recursion formulas

$$
x_{n+1}=\left\{N x_{n}+\theta\right\} \quad(n=0,1, \cdots)
$$

where $N=$ integer $>1$. These sequences have a long history in the literature and practice of computation; some early references are given by $\mathrm{O}$. Taussky and $\mathrm{J}$. Todd [6]. These sequences were shown to be equidistributed for almost all $x_{0}$ in [7]. Next we discuss "polynomial" sequences.

$$
x_{n}=\left\{n^{p} \alpha+c_{1} n^{p-1}+c_{2} n^{p-2}+\cdots+c_{p}\right\}, \quad \alpha \text { irrational. }
$$

H. Weyl [1] showed that these sequences are equidistributed. Finally we discuss the sequences $x_{n}=\left\{\theta^{n}\right\}$, which were shown by J. F. Koksma [3] to be equidistributed for almost all $\theta>1$.

Summary of results: The Weyl sequence $x_{n}=\{n \alpha\}, \alpha$ irrationai, is not equipartitioned by twos.

A Multiply sequence may fail to be equidistributed even if $x_{0}$ is transcendental.

Every equidistributed Multiply sequence is equipartitioned by twos.

Every sequence equidistributed by $k$ 's is equipartioned by $k$ 's.

Let $x_{1}, x_{2}, \cdots$ be any sequence in $C_{1}$ generated by a recurrence formula $x_{n+1}=F\left(x_{n}\right)$. This sequence cannot be equidistributed by $k$ 's for any $k>1$ if $F(x)$ has any point of continuity in $0<x<1$. In particular, no Multiply sequence is equidistributed by $k$ 's for any $k>1$.

Let $x_{1}, x_{2}, \cdots$ be an equidistributed sequence satisfying $x_{n+1}=\left\{N x_{n}\right\}, N=$ integer $>1$. Then

$$
\operatorname{Pr}\left(x_{n}>x_{n+1}>x_{n+2}\right)=\frac{1}{6}\left(1+N^{-1}\right) .
$$

Thus the sequence is not equipartitioned by $k$ 's for any $k \geqq 3$.

Let $\theta$ and $x_{0}$ be fixed. For each $N=2,3, \cdots$ form the Multiply sequence $x_{n}=x_{n}(N)$ from the recurrence formula (1.12). For almost all $x_{0}$ these sequences are equidistributed, and they are asymptotically completely equidistributed in this sense: For every positive integer $k$

$$
\lim _{N \rightarrow \infty} \operatorname{Pr}\left(a_{r} \leqq x_{n+r}(N)<b_{r} \text { for } r=0, \cdots, k-1\right)=\prod_{r=0}^{k-1}\left(b_{r}-a_{r}\right)
$$

if $0 \leqq a_{r}<b_{r} \leqq 1 \quad(r=0, \cdots, k-1)$. 
For almost all $x_{0}$ the Multiply sequence $x_{n}(N)$ is asymptotically completely equipartitioned in this sense: For every positive integer $k$

$$
\lim _{N \rightarrow \infty} \operatorname{Pr}\left(z^{(n)}(N) \in O_{j}\right)=\frac{1}{k !} \quad(j=1, \cdots, k !)
$$

where $O_{j}$ is any of the $k$ ! partitions of $C_{k}$, and where $z^{(n)}=\left(x_{n}, x_{n+1}, \cdots, x_{n+k-1}\right)$.

Let $f\left(z_{1}, \cdots, z_{k}\right)$ be any Riemann-integrable function in $C_{k}$ for which all the one-dimensional Riemann integrals over line segments in $C_{k}$ exist. Then for almost all starting values $x_{0}$ the Multiply sequences $x_{n}(N)$ have the limiting property

$$
\lim _{N \rightarrow \infty} \lim _{M \rightarrow \infty} \frac{1}{M} \sum_{n=1}^{M} f\left(x_{n}(N), \cdots, x_{n+k-1}(N)\right)=\int_{0}^{1} \cdots \int_{0}^{1} f\left(z_{1}, \cdots, z_{k}\right) d z_{1} \cdots d z_{k} .
$$

For almost all $x_{0}$ the Multiply sequence defined by (1.12) has the autocorrelation function

$$
R(\tau)=N^{-\tau}\left(\frac{1}{12}-\frac{1}{2}\{\beta\}+\frac{1}{2}\left\{\beta^{2}\right\}\right) \quad(\tau=0,1, \cdots)
$$

where $\beta=\left(N^{\tau}-1\right) \theta /(N-1)$. Thus

$$
-\frac{1}{24} N^{-\tau} \leqq R(\tau) \leqq \frac{1}{12} N^{-\tau} \quad(\tau=0,1, \cdots)
$$

so that $R(\tau) \rightarrow 0$ as $N \rightarrow \infty$ uniformly in $\tau$ for $\tau \neq 0$; in this sense the Multiply sequence $x_{n}(N)$ is asymptotically white.

Let $q(x)$. be a polynomial with real coefficients. Suppose that for some $x_{0}$ the sequence $x_{1}, x_{2}, \cdots$ generated by

$$
x_{n+1}=\left\{q\left(x_{n}\right)\right\} \quad(n=0,1, \cdots)
$$

is equidistributed in $0 \leqq x<1$. Then either $q(x)=x+\alpha$, $\alpha$ irrational, or $q(x)=$ $N x+\theta, \pm N=$ integer $>1$.

The "polynomial" sequence (1.13) of degree $p$ is equidistributed by $k$ 's if and only if $k \leqq p$.

Every polynomial sequence of degree $p \geqq 2$ is white.

The sequence $x_{n}=\left\{n^{2} \alpha\right\}, \alpha$ irrational, $0<\alpha<1$, is equipartitioned by threes if and only if $\alpha$ is one of the four numbers $(3 \pm \sqrt{3}) / 12,(9 \pm \sqrt{3}) / 12$.

The sequence $x_{n}=\left\{\theta^{n}\right\}$ is completely equidistributed for almost all $\theta>1$.

If $x_{n}=\left\{\theta^{n}\right\}$ is equidistributed by $r$ 's, then $\theta$ cannot be an algebraic number of degree $<r$. In particular, if $\left\{\theta^{n}\right\}$ is completely equidistributed, then $\theta$ is transcendental.

Every completely equidistributed sequence is white.

There is an equidistributed white sequence $x_{n}$ for which $\operatorname{Pr}\left(x_{n}>x_{n+1}\right)$ is not equal to $1 / 2$.

There is a sequence $x_{1}, x_{2}, \cdots$ equidistributed by twos for which the twodimensional derived sequence $\left(x_{2}, x_{3}\right),\left(x_{4}, x_{5}\right), \cdots$ is not equidistributed.

For any Multiply sequence $x_{n}$ the $r$-dimensional derived sequence $y^{(n)}=$ $\left(x_{n r}, x_{n r+1}, \cdots, x_{n r+r-1}\right)$ is not equidistributed in $C_{r}$ for any $r>1$.

The $r$-dimensional derived sequence $y^{(n)}$ formed from the polynomial sequence $x_{n}=\left\{\alpha n^{p}+\cdots+c_{p}\right\} \quad$ ( $\alpha$ irrational) is equidistributed by $k$ 's if and only if $k r \leqq p$. 
For almost all $\theta>1$, for every $r=1,2, \cdots$, the $r$-dimensional derived sequence $y^{(n)}$ formed from $x_{n}=\left\{\theta^{n}\right\}$ is completely equidistributed.

2. Weyl Sequences. Weyl showed [1] that $x_{n}=\{n \alpha\}$ is equidistributed if $\alpha$ is irrational. Let us compute $\operatorname{Pr}\left(x_{n}>x_{n+1}\right)$. We have $x>\{x+\alpha\}$ when $1-\alpha \leqq$ $x<1$. Therefore, by equidistribution,

$$
\operatorname{Pr}\left(x_{n}>x_{n+1}\right)=\operatorname{Pr}\left(1-\alpha \leqq x_{n}<1\right)=\alpha \neq \frac{1}{2} .
$$

As D. L. Jagerman has shown [5], Weyl sequences are not white. He has shown that, according to definition (1.9),

$$
R(\tau)=\frac{1}{12}-\int_{0}^{\alpha \tau}\left(\frac{1}{2}-\{u\}\right) d u \quad(\tau=0,1,2, \cdots) .
$$

Since the integral $\int_{0}^{x}\left(\frac{1}{2}-\{u\}\right) d u$ is a periodic function of $x$, we may also write

$$
\begin{aligned}
R(\tau) & =\frac{1}{12}-\int_{0}^{\{\alpha \tau\}}\left(\frac{1}{2}-u\right) d u \quad(\tau=0,12, \cdots) \\
& =\frac{1}{12}-\frac{1}{2}\{\alpha \tau\}+\frac{1}{2}\{\alpha \tau\}^{2} .
\end{aligned}
$$

Since the values $\{\alpha \tau\}$ are equidistributed in $(0,1)$, the sequence $R(\tau)$ takes values dense in the interval $\left(-\frac{1}{24}, \frac{1}{12}\right)$ for arbitrarily large integers $\tau$. Therefore, the spectral density $\phi(\omega)$ defined by (1.9) does not exist.

3. Multiply Sequences. Although Multiply sequences, defined by (1.2) with $N=$ integer $>1$, are equidistributed for almost all $x_{0}$ (c.f. [7]), it is not sufficient for equidistribution that $x_{0}$ be irrational.

Theorem 1. A Multiply sequence may fail to be equidistributed even if $x_{0}$ is transcendental.

Proof. Let $\theta=0$, and let $x_{0}$ be the Liouville number

$$
x_{0}=\sum_{\nu=1}^{\infty} N^{-\nu !} \text {. }
$$

This number is easily shown [9] to be transcendental. Then all the numbers $x_{n}$ have $N$-ary expansions beginning with .1 or with .0 ; in every case

$$
x_{n}=\left\{N^{n} x_{0}\right\}<N^{-1}+N^{-2}+N^{-3}<1 .
$$

Therefore, these numbers fail to be equidistributed.

Incidentally, it was Borel who first proved, by probabilistic arguments, that for almost all positive numbers $x_{0}<1$ the digits $0, \cdots, N-1$ appear with equal likelihood $1 / N$. The proof of equidistribution for $\theta \neq 0$ which appears in [7] follows from the Riesz ergodic theorem.

THEOREM 2. Every equidistributed Multiply sequence is equipartitioned by 2's.

Proof. We must show that the $x_{n}$ are distinct and that $\operatorname{Pr}\left(x_{n+1}>x_{n}\right)=1 / 2$ in the sense of the definition of "probability" given by (1.4).

The numbers $x_{n}$ are distinct because otherwise the sequence would ultimately 
be periodic and therefore not equidistributed. In other words, if $x_{p}=x_{q}$, then $x_{p+i}=x_{q+i}$ for all $i>0$.

Let $G$ be the set of numbers $x$ in $(0,1)$ with the property that $\{N x+\theta\}>x$. Since the $x_{n}$ are equidistributed, with $x_{n+1}=\left\{N x_{n}+\theta\right\}$, the theorem will be proved if it is shown that $G$ consists of a finite number of intervals whose lengths total $1 / 2$. Without loss of generality assume $0 \leqq \theta<1$. Then for $k=0,1, \cdots, N$

$$
\{N x+\theta\}=N x+\theta-k \text { for } x \text { in } E_{k}
$$

where $E_{0}, \cdots, E_{N}$ are the subintervals

$$
\begin{aligned}
& E_{0}: 0 \leqq x<\frac{1-\theta}{N} \\
& E_{k}: \frac{k-\theta}{N} \leqq x<\frac{k+1-\theta}{N} \quad(k=1, \cdots, N-1) \\
& E_{N}: \frac{N-\theta}{N} \leqq x<1 .
\end{aligned}
$$

Let $G_{k}$ be $G \cap E_{k}$, i.e., that portion of $G$ which lies in $E_{k}$. In $G_{k}$ we have $N x+$ $\theta-k>x$, or $x>(k-\theta) /(N-1)$. Since $(N-\theta) /(N-1)>1$, the set $G_{N}$ is empty; for $k<N$ the sets $G_{k}$ are the intervals

$$
\begin{aligned}
& G_{0}: 0 \leqq x<\frac{1-\theta}{N} \\
& G_{k}: \frac{k-\theta}{N-1}<x<\frac{k+1-\theta}{N} \quad(k=1, \cdots, N-1)
\end{aligned}
$$

since

$$
\frac{k-\theta}{N}<\frac{k-\theta}{N-1} \leqq \frac{k+1-\theta}{N} \quad(k=1, \cdots, N-1) .
$$

Therefore, $G$ is the union of the intervals $G_{0}, \cdots, G_{N-1}$, whose lengths total

$$
|G|=\sum_{k=0}^{N-1}\left|G_{k}\right|=\frac{1-\theta}{N}+\sum_{k=1}^{N-1}\left(\frac{k+1-\theta}{N}-\frac{k-\theta}{N-1}\right)
$$

for which an elementary computation gives the value $|G|=1 / 2$. This completes the proof.

This is a convenient context in which to prove the general result:

Theorem 3. Every sequence equidistributed by k's is equipartitioned by k's.

Proof. Given a sequence $x_{n}$ in the interval $C_{1}$ such that the vectors $z^{(n)}=\left(x_{n}, x_{n+1}, \cdots, x_{n+k-1}\right)$ are equidistributed in $C_{k}$, we must show that these vectors lie in the set $O_{j}$ with probability $1 / k$ ! where $O_{j}$ is any one of the $k$ ! subsets of $C_{k}$ :

$$
O_{1}: z_{1}<\cdots<z_{k}, \cdots, \quad O_{k}: z_{k}<\cdots<z_{1} .
$$

This is an immediate consequence of the well-known fact (see, for example, Koksma [4]) that

$$
\lim _{N \rightarrow \infty} \frac{1}{N} \sum_{n=1}^{N} f\left(z^{(n)}\right)=\int_{0}^{1} \cdots \int_{0}^{1} f(z) d z_{1} \cdots d z_{k}
$$


for all Riemann-integrable functions $f(z)=f\left(z_{1}, \cdots, z_{k}\right)$ if the sequence $z^{(n)}$ is equidistributed in $C_{k}$. In our case we simply define $f(z)=1$ if $z$ is in $O_{j}, f(z)=0$ otherwise. Then formula (3.6) reads:

$$
\operatorname{Pr}\left(z^{(n)} \text { in } O_{j}\right)=\text { volume of } O_{j} .
$$

By symmetry $O_{j}$ has volume $1 / k$ !.

Returning to Multiply sequences, we observe from the following general result that no Multiply sequence is equidistributed by $k$ 's for any $k>1$.

Theorem 4 . Let $x_{1}, x_{2}, \cdots$ be any sequence in $C_{1}$ generated by a recurrence formula $x_{n+1}=F\left(x_{n}\right)$. This sequence cannot be equidistributed by $k$ 's for any $k>1$ if $F(x)$ has any point of continuity in $0<x<1$.

Proof. Let $F(x)$ be continuous at $x=a$, with $F(a)=b$. Then there is a number $\delta>0$ such that $|F(x)-b|<1 / 4$ if $|x-a|<\delta$. Let $I$ be the intersection of $|x-a|<\delta$ with $0 \leqq x<1$; let $J$ be any interval in $C_{1}$ whose distance from $b$ exceeds $1 / 4$. Then no points $\left(x_{n}, x_{n+1}\right)$ lie in the rectangle $I \times J$. Therefore, the sequence $x_{n}$ is not equidistributed by twos, hence not equidistributed by $k$ 's for any $k>1$.

In the case of Multiply sequences, $F(x)=\{N x+\theta\}$, which is continuous at all but $N$ or $N-1$ points in $(0,1)$.

The question remains: Are Multiply sequences at least equipartitioned by $k$ 's for all $k$ ? We answer this question in the case $\theta=0$.

TheOREM 5. Let $x_{1}, x_{2}, \cdots$ be an equidistributed sequence satisfying $x_{n+1}=$ $\left\{N x_{n}\right\}$, where $N=$ integer $>1$. Then

$$
\operatorname{Pr}\left(x_{n}>x_{n+1}>x_{n+2}\right)=\frac{1}{6}\left(1+N^{-1}\right) .
$$

Thus, the sequence $x_{n}$ is not equipartitioned by $k$ 's for any $k \geqq 3$.

Proof. For $0 \leqq x<1$ define

$$
y=\{N x\}, \quad z=\{N y\}=\left\{N^{2} x\right\} .
$$

Let $G$ be the set of $x$ such that $x>y>z$. Since $x_{n}$ is equidistributed, it will suffice to show that $G$ is a collection of a finite number of intervals whose lengths total $\left(1+N^{-1}\right) / 6$. For this purpose we use the Borel interpretation of $y$ and $z$. We have

$$
x=\frac{A}{N}+\frac{y}{N}, \quad y=\frac{B}{N}+\frac{z}{N}
$$

where $A$ and $B$ are integers between 0 and $N-1$, and where $x, y$, and $z$ are $\geqq 0$ and $<1$. We proceed to enumerate the cases in which $x>y>z$. If $A=0$, then $x=y / N \leqq y$, so that the relation $x>y>z$ is impossible; in the same way we conclude that $B>0$. If $A=1, \cdots, N-1$ we have $x>y$ when

$$
\frac{A}{N}+\frac{y}{N}>y, \quad \text { or } \quad y<\frac{A}{N-1} .
$$

Similarly, $y>z$ when $z<B /(N-1)$. But $x>y$ implies $A \geqq B$, since

$$
x=\frac{A}{N}+\frac{y}{N}<\frac{A+1}{N} \leqq y \quad \text { if } \quad B \geqq A+1 .
$$

Therefore $x>y>z$ implies

$$
N-1 \geqq A \geqq B \geqq 1, \quad 0 \leqq z<B /(N-1) .
$$


Conversely, these inequalities imply

$$
y=\frac{B}{N}+\frac{z}{N}<\frac{A}{N}+\frac{1}{N} \cdot \frac{A}{N-1}=\frac{A}{N-1} .
$$

Therefore, by (3.11), the inequalities (3.13) imply $x>y$. Since the second half of (3.13) implies $y>z$, the inequalities (3.13) are sufficient as well as necessary for $x>y>z$. But each $x$ in $C_{1}$ has a unique $N$-ary representation

$$
x=\frac{A}{N}+\frac{B}{N^{2}}+\frac{z}{N^{2}} .
$$

Therefore, by (3.13) we see that the set $G=(x \mid x>y>z)$ is a collection of a finite number of intervals whose lengths total

$$
\begin{aligned}
|G| & =\sum_{A=1}^{N-1} \sum_{B=1}^{A} \frac{1}{N^{2}} \cdot \frac{B}{N-1} \\
& =\frac{1}{N^{2}(N-1)} \sum_{A=1}^{N-1} \frac{1}{2} A(A+1)=\frac{1}{6}\left(1+N^{-1}\right) .
\end{aligned}
$$

This completes the proof.

The preceding computation shows that, although $\operatorname{Pr}\left(x_{n}>x_{n+1}>x_{n+2}\right) \neq 1 / 6$, this number is approached as $N \rightarrow \infty$. In this sense, Multiply sequences with $\theta=0$ are asymptotically equipartitioned by 3 's. We shall show much more: Multiply sequences with any $\theta$ are asymptotically completely equidistributed as $N \rightarrow \infty$.

Theorem 6. Let $\theta$ and $x_{0}$ be fixed. For each $N=2,3, \cdots$ form the sequence $x_{n}=x_{n}(N)$ from the formula

$$
x_{n+1}=\left\{N x_{n}+\theta\right\} \quad(n=0,1,2, \cdots) .
$$

For almost all $x_{0}$ these sequences are all equidistributed, and

(3.18) $\lim _{N \rightarrow \infty} \operatorname{Pr}\left(a_{r} \leqq x_{n+r}(N)<b_{r}\right.$ for $\left.\quad r=0,1, \cdots, k-1\right)=\prod_{r=0}^{k-1}\left(b_{r}-a_{r}\right)$

if $0 \leqq a_{r}<b_{r} \leqq 1(r=0, \cdots, k-1)$. This result holds for all positive integers $k$.

Proof. This result follows from a calculation with Fourier series. For all real $x$ define the periodic function $\phi(x ; a, b)=\phi(x+1 ; a, b)$ such that

$$
\phi=1(a \leqq x<b) ; \quad \phi=0 \quad(0 \leqq x<a \quad \text { or } \quad b \leqq x<1) .
$$

If $0 \leqq z<b \leqq 1$ this function is discontinuous unless $a=0$ and $b=1$. For all sufficiently small $\epsilon>0$ we define continuous, periodic, piecewise linear functions $\phi^{+}(x ; a, b)$ and $\phi^{-}(x ; a, b)$ as follows: If $a=0$ and $b=1$, define $\phi^{+}=\phi^{-}=\phi \equiv 1$. If $a>0$ or $b<1$ define

$$
\begin{array}{ll}
\phi^{+}=\epsilon^{-1}(x-(a-\epsilon)) & (a-\epsilon \leqq x \leqq a) \\
\phi^{+}=1 & (a \leqq x \leqq b) \\
\phi^{+}=\epsilon^{-1}(b+\epsilon-x) & (b \leqq x \leqq b+\epsilon) \\
\phi^{+}=0 & (b+\epsilon \leqq x \leqq a-\epsilon+1) \\
\phi^{+}(x ; a, b) \equiv \phi^{+}(x+1 ; a, b) & \text { for all } x .
\end{array}
$$


Similarly define

$$
\begin{array}{ll}
\phi^{-}=\epsilon^{-1}(x-a) & (a \leqq x \leqq a+\epsilon) \\
\phi^{-}=1 & (a+\epsilon \leqq x \leqq b-\epsilon) \\
\phi^{-}=\epsilon^{-1}(b-x) & (b-\epsilon \leqq x \leqq b) \\
\phi^{-}=0 & (b \leqq x \leqq a+1) \\
\phi^{-}(x ; a, b) \equiv \phi^{-}(x+1 ; a, b) & \text { for all } x .
\end{array}
$$

Both functions $\phi^{+}$and $\phi^{-}$have uniformly and absolutely convergent Fourier series

$$
\phi^{ \pm}(x ; a, b)=\sum_{\nu} c_{\nu}^{ \pm}(a, b) \exp 2 \pi i \nu x \text { for all } x .
$$

Furthermore, for all $x$

$$
\phi^{-}(x ; a, b) \leqq \phi(x ; a, b) \leqq \phi^{+}(x ; a, b)
$$

and

$$
\int_{0}^{1} \phi^{ \pm}(x ; a, b) d x=c_{0}^{ \pm}(a, b)=b-a \pm \epsilon^{\prime}
$$

where $\epsilon^{\prime}=\epsilon$ unless $a=0$ and $b=1$, in which case $\epsilon^{\prime}=0$.

For each integer $N>1$ the sequence $x_{n}$ is equidistributed for almost all $x_{0}$. Therefore, since the set of $N$ 's is denumerable, all sequences $x_{n}{ }^{(N)}$ are equidistributed for almost all $x_{0}$; in the rest of the proof we assume $x_{0}$ to have any value such that all the sequences $x_{n}{ }^{(N)}$ are equidistributed.

A trivial inductive proof shows that for each $n=0,1, \ldots$

$$
x_{n+r} \equiv N^{r} x_{n}+\theta_{r}(\bmod 1) \quad(r=0,1, \cdots)
$$

where $\theta_{r}=\left(N^{r}-1\right) \theta /(N-1)$. Therefore, $a_{r} \leqq x_{n+r}<b_{r}$ for $r=0, \cdots$, $k-1$ if and only if

$$
\prod_{r=0}^{k-1} \phi\left(N^{r} x_{n}+\theta_{r} ; a_{r}, b_{r}\right)=1
$$

By the equidistribution of $x_{n}$

$$
\begin{aligned}
\operatorname{Pr}\left(a_{r}\right. & \left.\leqq x_{n+r}<b_{r} \text { for } r=0,1, \cdots, k-1\right) \\
& =\lim _{M \rightarrow \infty} M^{-1} \sum_{n=1}^{M} \prod_{r=0}^{k-1} \phi\left(N^{r} x_{n}+\theta_{r} ; a_{r}, b_{r}\right) \\
& =\int_{0}^{1} \prod_{r=0}^{k-1} \phi\left(N^{r} x+\theta_{r} ; a_{r}, b_{r}\right) d x .
\end{aligned}
$$

Denoting this probability by $P$, we find from the inequalities (3.23)

$$
\int_{0}^{1} \prod_{r=0}^{k-1} \phi^{-}\left(N^{r} x+\theta_{r} ; a_{r}, b_{r}\right) d x \leqq P \leqq \int_{0}^{1} \prod_{r=0}^{k-1} \phi^{+}\left(N^{r} x+\theta_{r} ; a_{r}, b_{r}\right) d x .
$$

Using the Fourier series (3.22) for $\phi^{+}$, we find

$$
P \leqq \int_{0}^{1} \prod_{r=0}^{k-1} \sum_{\nu_{r}} c_{\nu_{r}}^{+}\left(a_{r}, b_{r}\right) \exp 2 \pi i \nu_{r}\left(N^{r} x+\theta_{r}\right) d x
$$


Term-by-term integration gives

$$
P \leqq \sum_{\nu_{0}} \cdots \sum_{\nu_{k-1}} c_{\nu_{0}}^{+}\left(a_{0}, b_{0}\right) \cdots c_{\nu_{k-1}}^{+}\left(a_{k-1}, b_{k-1}\right) I_{\nu}(N)
$$

where

$$
I_{\nu}(N)=\omega_{\nu} \int_{0}^{1} \exp \left(2 \pi i\left(\nu_{0}+\nu_{1} N+\cdots+\nu_{k-1} N^{k-1}\right) x\right) d x
$$

where $\omega_{\nu}=\exp 2 \pi i \sum \nu_{r} \theta_{r}$. Unless all $\nu_{r}=0$ the integer $\nu_{0}+\nu_{1} N+\cdots+\nu_{k-1} N^{k-1}$ is non-zero for all sufficiently large $N$, and $I_{\nu}(N)=0$. Thus $I_{\nu}(N) \rightarrow 0$ as $N \rightarrow \infty$ for each non-zero lattice point $\nu=\left(\nu_{0}, \cdots, \nu_{k-1}\right)$. Since all the Fourier series for the functions $\phi^{+}\left(N^{r} x+\theta_{r} ; a_{r}, b_{r}\right)$ converge uniformly with respect to $N$ and $x$, we may take the limit of the sum in (3.30) as $N \rightarrow \infty$ term-by-term to obtain

$$
\underset{N \rightarrow \infty}{\limsup } P \leqq c_{0}^{+}\left(a_{0}, b_{0}\right) \cdots c_{0}^{+}\left(a_{k-1}, b_{k-1}\right) \text {. }
$$

From the identity (3.24) we conclude

$$
\limsup _{N \rightarrow \infty} P \leqq \prod_{r=0}^{k-1}\left(b_{r}-a_{r}+\epsilon_{r}^{\prime}\right) .
$$

Working with $\phi^{-}$instead of $\phi^{+}$, we find similarly

$$
\liminf _{N \rightarrow \infty} P \geqq \prod_{r=0}^{k-1}\left(b_{r}-a_{r}-\epsilon_{r}^{\prime}\right) .
$$

Since $\epsilon_{r}^{\prime} \leqq \epsilon$ is arbitrarily small, we obtain the required result $P \rightarrow \prod\left(b_{r}-a_{r}\right)$ as $N \rightarrow \infty$.

From this result we can show that for almost all starting values $x_{0}$ the Multiply sequences $\dot{x}_{n}(N)$ are asymptotically completely equipartitioned as $N \rightarrow \infty$.

TheOREM 7. For almost all $x_{0}$ the Multiply sequences $x_{n}(N)$ defined by (3.17) have the property

$$
\lim _{N \rightarrow \infty} \operatorname{Pr}\left(z^{(n)}(N) \text { in } O_{j}\right)=1 / k !
$$

where $O_{j}$ is any of the $k$ ! partitions (3.5) of $C_{k}$, and where $z^{(n)}(N)=\left(x_{n}(N), \cdots\right.$, $\left.x_{n+k-1}(N)\right)$.

Proof. Let $f\left(z_{1}, \cdots, z_{k}\right)=1$ if $z$ lies in $O_{j}, f(z)=0$ otherwise. By the equidistribution of each sequence $x_{n}(N)$, we have the existence of the limit

$$
\begin{aligned}
\int_{0}^{1} f\left(x,\left\{N x+\theta_{1}\right\}\right. & \left., \cdots,\left\{N^{k-1} x+\theta_{k-1}\right\}\right) d x \\
& =\lim _{M \rightarrow \infty} \frac{1}{M} \sum_{n=1}^{M} f\left(x_{n},\left\{N x_{n}+\theta_{1}\right\}, \cdots,\left\{N^{k-1} x_{n}+\theta_{k-1}\right\}\right) d x \\
& =\lim _{M \rightarrow \infty} \frac{1}{M} \sum_{n=1}^{M} f\left(z^{(n)}(N)\right)=\operatorname{Pr}\left(z^{(n)}(N) \text { in } O_{j}\right)
\end{aligned}
$$

where the numbers $\theta_{r}$ are defined in (3.25). Let $\epsilon>0$ be given. Since $f(z)$ is Riemann-integrable in $C_{k}$, we can partition $C_{k}$ into $k$-dimensional boxes $B_{v}$ with volumes $\Delta V_{\nu}$ such that

$$
\sum_{\nu} M_{\nu} \Delta V_{\nu}-\epsilon \leqq \int \cdots \int_{C_{k}} f(z) d z_{1} \cdots d z_{k} \leqq \sum_{\nu} m_{\nu} \Delta V_{\nu}+\epsilon
$$


where $m_{\nu} \leqq f(z) \leqq M_{\nu}$ for $z$ in $B_{\nu}$. Let $P_{\nu}(N ; M)$ be $1 / M$ times the number of points $z^{(1)}(N), \cdots, z^{(M)}(N)$ which lie in $B_{\nu}$. Then

$$
\sum_{\nu} m_{\nu} P_{\nu}(N ; M) \leqq \frac{1}{M} \sum_{n=1}^{M} f\left(z^{(n)}(N)\right) \leqq \sum_{\nu} M_{\nu} P_{\nu}(N ; M) .
$$

By the last theorem we know that $P_{\nu}(N ; M)$ tends to a limit $P_{\nu}(N)$ as $M \rightarrow \infty$ (the form of this limit is given by the integral in (3.27)) and that $P_{\nu}(N)$ tends to the limit $\Delta V_{\nu}$ as $N \rightarrow \infty$. Therefore, by (3.35)

$$
\sum_{\nu} m_{\nu} P_{\nu}(N) \leqq \operatorname{Pr}\left(z^{(n)}(N) \text { in } O_{j}\right) \leqq \sum_{\nu} M_{\nu} P_{\nu}(N)
$$

and

$$
\begin{aligned}
& \limsup _{N \rightarrow \infty} \operatorname{Pr}\left(z^{(n)}(N) \text { in } O_{j}\right) \leqq \sum_{\nu} M_{\nu} \Delta V_{\nu} \\
& \liminf _{N \rightarrow \infty} \operatorname{Pr}\left(z^{(n)}(N) \text { in } O_{j}\right) \geqq \sum_{\nu} m_{\nu} \Delta V_{\nu} .
\end{aligned}
$$

Using (3.34) and letting $\epsilon \rightarrow 0$ we obtain the existence of the limit

$$
\begin{aligned}
\lim _{N \rightarrow \infty} \operatorname{Pr}\left(z^{(n)}(N) \text { in } O_{j}\right) & =\int_{C_{k}} f(z) d z \\
& =\text { volume of } O_{j}=1 / k !
\end{aligned}
$$

This completes the proof.

We have, in fact, proved the more general result:

THEOREM 8. Let $f\left(x_{1}, \cdots, x_{k}\right)$ be any Riemann-integrable function in $C_{k}$ for which all the one-dimensional Riemann integrals (3.33) over line segments in $C_{k}$ exist. Then for almost all starting values $x_{0}$ the Multiply sequences $x_{n}(N)$ defined by (3.17) have the limiting property

$$
\begin{aligned}
\lim _{N \rightarrow \infty} \lim _{M \rightarrow \infty} \frac{1}{M} \sum_{n=1}^{M} f\left(x_{n}(N), \cdots, x_{n+k-1}(N)\right) & \\
& =\int_{0}^{1} \cdots \int_{0}^{1} f\left(z_{1}, \cdots, z_{k}\right) d z_{1} \cdots d z_{k} .
\end{aligned}
$$

Next we compute the autocorrelation function (1.9) of a Multiply sequence.

Theorem 9. For almost all $x_{0}$ the sequence defined by $x_{n+1}=N x+\theta, N=$ integer $>1$, has autocorrelation function

$$
R(\tau)=N^{-\tau}\left(\frac{1}{12}-\frac{1}{2}\{\beta\}+\frac{1}{2}\{\beta\}^{2}\right) \quad(\tau=0,1, \cdots)
$$

where $\beta=\left(N^{\tau}-1\right) \theta /(N-1)$. Thus

$$
-\frac{1}{24} N^{-\tau} \leqq R(\tau) \leqq \frac{1}{12} N^{-\tau} \quad(\tau=0,1, \cdots)
$$

so that $R(\tau) \rightarrow 0$ as $N \rightarrow \infty$ uniformly in $\tau$ for $\tau \neq 0$.

Proof. We use Jagerman's Theorem (1.9), (1.10). We have

$$
F(\tau, k, \nu)=\lim _{M \rightarrow \infty} \frac{1}{2 M} \sum_{j-1}^{M}\left(\cos 2 \pi z_{j}^{-}-\cos 2 \pi z_{j}^{+}\right)
$$


where $z_{j}^{ \pm}=k x_{j} \pm x_{j+\tau}$. Letting “”” mean "congruent modulo 1 ", we find from

$$
\begin{aligned}
z_{j}^{ \pm} & \equiv k\left(N^{j} x_{0}+\frac{N^{j}-1}{N-1} \theta\right) \pm \nu\left(N^{j+\tau} x_{0}+\frac{N^{j+\tau}-1}{N-1} \theta\right) \\
& \equiv N^{j} x_{0}{ }^{\prime}+\frac{N^{j}-1}{N-1} \theta^{\prime}+\nu \beta
\end{aligned}
$$

where

$$
x_{0}^{\prime}=\left(k \pm \nu N^{\tau}\right) x_{0}, \quad \theta^{\prime}=(k \pm \nu N) \theta, \quad \beta= \pm \frac{N^{\tau}-1}{N-1} \theta .
$$

From (3.42) we observe, again using (3.25), that $z_{j}^{ \pm} \equiv x_{j}{ }^{\prime}+\nu \beta$, where $x_{j}{ }^{\prime}$ is the Multiply sequence satisfying

$$
x_{j+1}^{\prime} \equiv\left\{N x_{j}^{\prime}+\theta^{\prime}\right\} \quad(j=0,1, \cdots) .
$$

The numbers $x_{j}{ }^{\prime}$ are equidistributed for almost all $x_{0}{ }^{\prime}$, hence by (3.43) equidistributed for almost all $x_{0}$ if $k \pm \nu N^{r} \neq 0$. By Weyl's criterion the translates $z_{j} \equiv x_{j}{ }^{\prime}$ $+\nu \beta$ are equidistributed when the $x_{j}^{\prime}$ are equidistributed. Therefore,

$$
\lim _{M \rightarrow \infty} \frac{1}{M} \sum_{j=1}^{M} \cos 2 \pi z_{j}^{ \pm}=\int_{0}^{1} \cos 2 \pi z d z=0
$$

for almost all $x_{0}$ if the integer $k \pm \nu N^{\tau} \neq 0$. But $k+\nu N^{\tau} \geqq 2$ for all positive $k$ and $\nu$. Therefore, by (3.41),

$$
\begin{aligned}
& F(\tau, k, \nu)=0 \text { unless } k-\nu N^{\tau}=0 \\
& F\left(\tau, \nu N^{\tau}, \nu\right)=\frac{1}{2} \cos 2 \pi \nu \beta .
\end{aligned}
$$

The last identity follows from $z_{j}^{-}=\nu \beta$ for all $j$ if $k=\nu N^{\tau}$. By Jagerman's theorem (1.11) and by (3.46)

$$
\begin{aligned}
R(\tau) & =\sum_{k=1}^{\infty} \sum_{\nu=1}^{\infty} \frac{F(\tau, k, \nu)}{\pi^{2} k \nu} \\
& =\sum_{\nu=1}^{\infty} \frac{\cos 2 \pi \nu \beta}{2 \pi^{2} \nu^{2} N^{\tau}} .
\end{aligned}
$$

Using the well-known identity

$$
\sum_{\nu=1}^{\infty} \frac{\cos 2 \pi \nu x}{\pi^{2} \nu^{2}}=\frac{1}{6}-x+x^{2} \quad(0 \leqq x \leqq 1)
$$

we obtain the required result (3.40).

We should like also to compute the spectral density

$$
\begin{aligned}
\phi(\omega) & =R(0)+2 \sum_{\tau=1}^{\infty} R(\tau) \cos 2 \pi \tau \omega \\
& =\frac{1}{12}+\sum_{\tau=1}^{\infty} N^{-\tau}\left(\frac{1}{6}-\left\{\frac{N^{\tau}-1}{N-1} \theta\right\}+\left\{\frac{N^{\tau}-1}{N-1} \theta\right\}^{2}\right) \cos 2 \pi \tau \omega .
\end{aligned}
$$


If $\theta=0$, i.e., if $x_{n}=\left\{N^{n} x_{0}\right\}$, this series may be summed to the value

$$
\begin{aligned}
\phi(\omega) & =\frac{1}{12}+\sum_{\tau=1}^{\infty} N^{-\tau}\left(\frac{1}{6}\right) \cos 2 \pi \tau \omega \\
& =-\frac{1}{12}+\frac{1}{6} \operatorname{Re}\left(1-N^{-1} e^{2 \pi i \tau \omega}\right)^{-1} \\
\phi(\omega) & =\frac{1}{12} \frac{1-N^{-2}}{1-2 N^{-1} \cos 2 \pi \omega+N^{-2}} .
\end{aligned}
$$

Multiply sequences and Weyl sequences are generated by recurrence formulas $x_{n+1}=\left\{p\left(x_{n}\right)\right\}$, where $p(x)$ is a polynomial of first degree. In the next theorem we assert that there are no polynomials of higher degree which generate equidistributed sequences.

Theorem 10. Let $p(x)$ be a polynomial with real coefficients. Suppose that for some $x_{0}$ the sequence $x_{1}, x_{2}, \cdots$ generated by

$$
x_{n+1}=\left\{p\left(x_{n}\right)\right\} \quad(n=0,1, \cdots)
$$

is equidistributed in $0 \leqq x<1$. Then either $p(x)=x+\alpha, \alpha$ irrational, or $p(x)=$ $N x+\theta, \pm N=$ integer $>1$.

Proof. For any assertion $S_{n}$ we have $\operatorname{Pr}\left(S_{n}\right)=\operatorname{Pr}\left(S_{n+1}\right)$, since the number of numbers $n=1, \cdots, M$ for which $S_{n}$ is true differs by at most 1 from the corresponding number for $S_{n+1}$. In particular,

$$
\begin{aligned}
\operatorname{Pr}\left(x_{n}<y\right) & =\operatorname{Pr}\left(x_{n+1}<y\right) \\
& =\operatorname{Pr}\left(\left\{p\left(x_{n}\right)\right\}<y\right)
\end{aligned}
$$

or, by equidistribution, if $0<y<1$,

$$
y=m(x \mid\{p(x)\}<y, \quad 0<x<1),
$$

i.e., $y$ is the measure of the finite collection of intervals of values $x$ such that $\{p(x)\}<$ $y, 0<x<1$. There is at most a finite number of points $z_{i}$ such that $p^{\prime}\left(z_{i}\right)=0$. Let $y=b$ be any point satisfying

$$
0<b<1, \quad b \neq\{p(0)\}, \quad b \neq\{p(1)\}, \quad b \neq\left\{p\left(z_{i}\right)\right\} \quad \text { for any } z_{i} .
$$

Let $x=a_{1}, a_{2}, \cdots, a_{k}$ be the finite collection of points in the open interval $(0,1)$ such that $b=\left\{p\left(a_{j}\right)\right\}$. There must be at least one such point $a_{1}$, for otherwise there would be an interval of values surrounding $b$ which were not achieved by $p(x)$; then none of the values $x_{1}, x_{2}, \cdots$ could be in this interval, and the sequence $x_{n}$ could not be equidistributed. Let

$$
N_{j}=\left[p\left(a_{j}\right)\right], \quad b=p\left(a_{j}\right)-N_{j} \quad(j=1, \cdots, k) .
$$

Since $p^{\prime}\left(a_{j}\right) \neq 0$, there are inverse functions $\xi_{j}(y)$ uniquely defined in a neighborhood $|y-b|<\delta$ such that

$$
\begin{aligned}
\xi_{j}(b) & =a_{j}, \quad y=\left\{p\left(\xi_{j}(y)\right\}=p\left(\xi_{j}(y)\right)-N_{j},\right. \\
p^{\prime}\left(x_{j}\right) & \neq 0, \quad 0<\xi_{j}(y)<1 \quad(j=1, \cdots, k) .
\end{aligned}
$$


For $|y-b|<\delta$ differentiation of the identity (3.52) gives

$$
\begin{aligned}
1 & =\frac{d}{d y} m(x \mid\{p(x)\}<y, \quad 0<x<1) \\
& =\sum_{j=1}^{k}\left|\frac{d \xi_{j}}{d y}\right|=\sum_{j=1}^{k} \theta_{j} \frac{d \xi_{j}(y)}{d y}
\end{aligned}
$$

where $\theta_{j}=\operatorname{sgn} \xi^{\prime}(b)=\operatorname{sgn} p^{\prime}\left(a_{j}\right)= \pm 1$.

We now consider each function $\xi_{j}(y)$ in the whole complex $y$-plane. This function is analytic except for branch points $y_{j \nu}=p\left(z_{\nu}\right)-N_{j}$ where $z_{1}, z_{2}, \cdots$ are the zeros of $p^{\prime}(z)$. For $j=1, \cdots, k$ there are at most $k(d-1)$ distinct points $y_{j v}$ if $d$ is the degree of $p(z)$. Let the $y$-plane be cut by non-intersecting curves extending from the points $y_{j \nu}$ to the point at infinity. In the cut plane every function $\xi_{j}(y)$ is a uniquely-defined analytic function of $y$ satisfying

$$
y=p\left(\xi_{j}(y)\right)-N_{j} \quad(j=1, \cdots, k) .
$$

Analytic continuation of (3.56) gives

$$
1=\sum_{j=1}^{k} \theta_{j} \frac{d \xi_{j}(y)}{d y}=\sum_{j=1}^{k} \frac{\theta_{j}}{p^{\prime}\left(\xi_{j}(y)\right)}
$$

in the whole cut plane. Let $y$ tend to infinity in the cut plane. By (3.57) each point $\xi_{j}(y)$ tends to infinity because $d=$ degree of $p \geqq 1$. If $d>1$, then (3.58) gives a contradiction, since $\xi_{j}(y) \rightarrow \infty$ would imply $p^{\prime}\left(\xi_{j}(y)\right) \rightarrow \infty$ and $1=0$ in the limit.

Therefore $d=1$, and $p(x)=A x+B, A \neq 0$. Then $\theta_{j}=\operatorname{sgn} \xi_{j}^{\prime}(b)=\operatorname{sgn} A$. Equation (3.58) now yields $1=k / A, A= \pm$ the positive integer $k$. The case $A=-1$ is impossible because $x_{n+1}=\left\{-x_{n}+B\right\}$ gives the two-valued sequence

$$
x_{2 m}=\left\{x_{0}\right\}, \quad x_{2 m+1}=\left\{-x_{0}+B\right\} \quad(m=0,1, \cdots) .
$$

If $A=1$ we must have $B$ equal to an irrational number $\alpha$; otherwise $x_{n}$ is periodic. If $k=2,3, \cdots$, the proof is completed by setting $N=A= \pm k, \theta=B$.

With regard to sequences generated by $x_{n+1}=\left\{N x_{n}+\theta\right\}$ where $N=$ integer $<$ -1 , the argument in [7] proving that $x_{n}$ is equidistributed for almost all $x_{0}$ was made only for $N=$ integer $>1$; but the proof also holds for $N=$ integer $<-1$.

4. Polynomial Sequences. Weyl [1] proved that for any integer $p>0$ the sequence

$$
x_{n}=\left\{\alpha n^{p}+c_{1} n^{p-1}+c_{2} n^{p-2}+\cdots+c_{p}\right\} \quad(n=0,1, \cdots)
$$

is equidistributed in $C_{1}^{\prime}$ if $\alpha$ is irrational. We shall study some sequential properties of these "polynomial" sequences.

Theorem 11. If the leading coefficient $\alpha$ is irrational, a polynomial sequence (4.1) of degree $p$ is equidistributed by $p$ 's but is not equidistributed by $(p+1)$ 's.

Remark. A sequence $x_{n}$ equidistributed by $r$ 's is equidistributed by $j$ 's for all $j<r$. This follows directly from the definition (1.2) of equidistribution if we set $z_{i}{ }^{(n)}=x_{r+i-1} \quad(i=1, \cdots, r), \quad a_{j+1}=\cdots=a_{r}=0, \quad b_{j+1}=\cdots=b_{r}=1$. Therefore, Theorem 11 implies that the polynomial sequence (4.1) is equidistributed by $k$ 's if and only if $k \leqq p$. 
Proof. First we show that $x_{n}$ is not equidistributed by $(p+1)$ 's. Let $f(n)=$ $\alpha n^{p}+\cdots+c_{p}$. Let $\Delta$ be the forward-difference operator: $\Delta f(n)=f(n+1)-$ $f(n)$. Then

$$
\Delta^{p} f(n)=p ! \alpha
$$

or

$$
k_{0} f(n)+k_{1} f(n+1)+\cdots+k_{p} f(n+p)=p ! \alpha
$$

where $k_{\nu}$ is the constant

$$
k_{\nu}=(-1)^{p-\nu}\left(\begin{array}{l}
p \\
\nu
\end{array}\right) \quad(\nu=0,1, \cdots, p) .
$$

For this choice of the integers $k_{\nu}$ we have, for all $N$,

$$
\begin{aligned}
\frac{1}{N} \sum_{n=0}^{N-1} \exp 2 \pi i\left(k_{0} x_{n}+k_{1} x_{n+1}+\cdots+k_{p} x_{n+p}\right) \\
=\frac{1}{N} \sum_{n=0}^{N-1} \exp 2 \pi i\left(k_{0} f(n)+\cdots+k_{p} f(n+p)\right)=\exp 2 \pi i p ! \alpha \neq 0 .
\end{aligned}
$$

Therefore, by the Weyl criterion, the vectors

$$
Z^{(n)}=\left(x_{n}, x_{n+1}, \cdots, x_{n+p}\right)
$$

are not equidistributed in $C_{p+1}$, i.e., the sequence $x_{n}$ is not equidistributed by $(p+1)$ 's.

However, if $k_{0}, \cdots, k_{p-1}$ are any $p$ integers not all zero, we have

$$
\sum_{\nu=0}^{p-1} k_{\nu} f(n+\nu)=\sum_{\nu=0}^{p-1} k_{\nu}{ }^{\prime} \Delta^{\nu} f(n)
$$

where

$$
k_{\nu}^{\prime}=\left(\begin{array}{l}
\nu \\
\nu
\end{array}\right) k_{\nu}+\left(\begin{array}{c}
\nu+1 \\
\nu
\end{array}\right) k_{\nu+1}+\cdots+\left(\begin{array}{c}
p-1 \\
\nu
\end{array}\right) k_{p-1} .
$$

The integers $k_{\nu}{ }^{\prime}$ are not all zero; in fact, $k_{s}{ }^{\prime}=k_{s}$ if $\nu=s$ is the largest integer $\leqq$ $p-1$ such that $k_{\nu} \neq 0$. Let $\nu=r$ be the smallest integer such that $k_{\nu}{ }^{\prime} \neq 0$. Then the polynomial (4.2) is a polynomial of degree $p-r \geqq 1$ with irrational leading coefficient

$$
\beta=p(p-1) \cdots(p-r+1) \alpha k_{r}^{\prime} .
$$

Then the polynomial (4.2) has the form $\beta n^{p-r}+\cdots$, and by the equidistribution of polynomials $(\bmod 1)$ with irrational leading coefficients

$$
\frac{1}{N} \sum_{n=0}^{N-1} \exp 2 \pi i\left(\beta n^{p-r}+\cdots\right) \rightarrow 0 \text { as } N \rightarrow \infty
$$

or, since $x_{\nu}=\{f(\nu)\}$,

$$
\frac{1}{N} \sum_{n=0}^{N-1} \exp 2 \pi i\left(k_{0} x_{n}+k_{1} x_{n+1}+\cdots+k_{n+p-1} x_{n+p-1}\right) \rightarrow 0 .
$$


Therefore, the vectors $z^{(n)}=\left(x_{n}, \cdots, x_{n+p-1}\right)$ are equidistributed in $C_{p}$, i.e., the sequence $x_{n}$ is equidistributed by $p$ 's.

By using the identity (1.11), Jagerman [5] showed that the sequence $x_{n}=\left\{n^{2} \alpha\right\}$ is white. Without using Jagerman's identity, one can derive in an elementary way the general result:

Theorem 12. If $p \geqq 2$ every sequence $x_{n}=\left\{\alpha n^{p}+c_{1} n^{p-1}+\cdots+c_{p}\right\}$, $\alpha$ irrational, is white.

Proof. Let $\tau$ be any positive integer. For every integer $k_{0}, k_{1}$ not both zero, we have, as in the preceding proof,

$k_{0}\left(\alpha n^{p}+c_{1} n^{p-1}+\cdots\right)+k_{1}\left(\alpha(n+\tau)^{p}+c_{1}(n+\tau)^{p-1}+\cdots\right)=$ polynomial in $n$ of degree $\geqq 1$ with irrational leading coefficient.

Therefore, the vectors $\left(x_{n}, x_{n+\tau}\right) \quad(n=0,1, \cdots)$ are equidistributed in $C_{2}$. Therefore,

$$
\begin{aligned}
R(\tau) & =\lim _{N \rightarrow \infty} \frac{1}{N} \sum_{n=0}^{N-1}\left(x_{n}-\frac{1}{2}\right)\left(x_{n+\tau}-\frac{1}{2}\right) \\
& =\int_{0}^{1} \int_{0}^{1}\left(x-\frac{1}{2}\right)\left(y-\frac{1}{2}\right) d x d y=0 .
\end{aligned}
$$

We know from Theorem 11 that no choice of $\alpha$ will make the sequence $x_{n}=\left\{n^{2} \alpha\right\}$ equidistributed by threes. Can we nevertheless choose $\alpha$ so as to make this sequence equipartioned by threes?

TheOREM 13. The sequence $x_{n}=\left\{n^{2} \alpha\right\}, \alpha$ irrational, $0<\alpha<1$, is equipartitioned by threes if and only if $\alpha$ is one of the four numbers $(3 \pm \sqrt{ } \overline{3}) / 12,(9 \pm \sqrt{3}) / 12$.

Proof. First we shall find values $\alpha$ which make

$$
\operatorname{Pr}\left(x_{n}>x_{n+1}>x_{n+2}\right)=\frac{1}{6} .
$$

An acceptable value $\alpha$ must also make the five other orderings of $x_{n}, x_{n+1}, x_{n+2}$ occur with probability $1 / 6$. In the unit cube $C_{3}$ we define the characteristic function $\phi(x, y, z)$ of the set $x>y>z: \phi=1$ for points in the set, $\phi=0$ for other points in $C_{3}$. The relation (4.6) is equivalent to the existence of the limit

$$
\lim _{N \rightarrow \infty} \frac{1}{N} \sum_{n=1}^{N} \phi\left(x_{n}, x_{n+1}, x_{n+2}\right)=\frac{1}{6}
$$

The function $\phi(x, y, z)$ has a Fourier series:

$$
\phi(x, y, z) \sim \sum_{p, q, r} c_{p q r} \exp 2 \pi i(p x+q y+r z) .
$$

If $\phi$ is defined by periodicity outside $C_{3}$, this series converges to the piecewise constant function $\phi$ except at the points of discontinuity. In particular, since the numbers $x_{\nu}=\left\{\nu^{2} \alpha\right\}, \alpha$ irrational, are distinct positive numbers $<1$, we have

$$
\begin{aligned}
\phi\left(x_{n}, x_{n+1}, x_{n+2}\right) & =\sum_{p, q, r} c_{p q r} \exp 2 \pi i\left(p x_{n}+q x_{n+1}+r x_{n+2}\right) \\
& =\sum_{p, q, r} c_{p q r} \exp 2 \pi i\left(p n^{2}+q(n+1)^{2}+r(n+2)^{2}\right) \alpha .
\end{aligned}
$$


Observing that $p n^{2}+q(n+1)^{2}+r(n+2)^{2}$ is independent of $n$ if and only if $p, q, r$ are proportional to $1,-2,1$, we compute

(4.10) $\lim _{N \rightarrow \infty} \frac{1}{N} \sum_{n=1}^{N} \exp \left(p x_{n}+q x_{n+1}+r x_{n+2}\right)=\left\{\begin{array}{l}e^{4 \pi i r \alpha} \text { if } p=r, q=-2 r \\ 0 \text { otherwise. }\end{array}\right.$

If we can justify the interchange of limits

$$
\begin{aligned}
\lim _{\mathrm{v} \rightarrow \infty} \frac{1}{N} \sum_{n=1}^{N} \sum_{p, q, r} c_{p, q, r} \exp 2 \pi i\left(p x_{n}+q x_{n+1}+r x_{n+2}\right) \\
=\sum_{p, q, r} c_{p q r} \lim _{N \rightarrow \infty} \frac{1}{N} \sum_{n=1}^{N} \exp 2 \pi i\left(p x_{n}+q x_{n+1}+r x_{n+2}\right)
\end{aligned}
$$

we shall find, by (4.10),

$$
\lim _{N \rightarrow \infty} \frac{1}{N} \sum_{n=1}^{N} \phi\left(x_{n}, x_{n+1}, x_{n+2}\right)=\sum_{r=-\infty}^{\infty} c_{r} e^{4 \pi i r \alpha}
$$

where $c_{r}=c_{r,-2 r, r}$. We compute the Fourier coefficient

$$
\begin{aligned}
& c_{r}=\int_{0}^{1} \int_{0}^{1} \int_{0}^{1} \phi(x, y, z) e^{-2 \pi i r(x-2 y+z)} d x d y d z \\
& c_{r}=\int_{0}^{1} \int_{0}^{x} \int_{0}^{y} e^{-2 \pi i r(x-2 y+z)} d z d y d x \\
& c_{r}=\frac{1}{4 \pi^{2} r^{2}}(r \neq 0), c_{0}=\frac{1}{6} .
\end{aligned}
$$

Then formula (4.12) gives

$$
\lim _{N \rightarrow \infty} \frac{1}{N} \sum_{n=1}^{N} \phi\left(x_{n}, x_{n+1}, x_{n+2}\right)=\frac{1}{6}+\sum_{r=1}^{\infty} \frac{\cos 4 \pi r \alpha}{2 \pi^{2} r^{2}} .
$$

The series on the right has the sum

$$
\begin{array}{ll}
S(\alpha)=\frac{1}{4}-\alpha+2 \alpha^{2} & \left(0 \leqq \alpha \leqq \frac{1}{2}\right) \\
S(\alpha)=S\left(\alpha-\frac{1}{2}\right) & \left(\frac{1}{2} \leqq \alpha \leqq 1\right) .
\end{array}
$$

We require those values of $\alpha$ which make $S(\alpha)=1 / 6$. Solving the quadratic equation, we find

$$
\alpha=(3 \pm \sqrt{3}) / 12 \quad\left(0<\alpha<\frac{1}{2}\right), \quad \alpha=(9 \pm \sqrt{3}) / 12 \quad\left(\frac{1}{2}<\alpha<1\right) .
$$

If the interchange of limits (4.11) is justified, we have shown that $\operatorname{Pr}\left(x_{n}>x_{n+1}>\right.$ $\left.x_{n+2}\right)=1 / 6$ if and only if $\alpha$ is one of the values (4.16).

Now we shall justify the interchange of limits. The Fourier series (4.8) has partial sums

$$
s_{\underline{m}}(x, y, z)=s_{m_{1}, m_{2}, m_{3}}(x, y, z)=\sum_{|p| \leqq m_{1}} \sum_{|q| \leqq m_{2}} \sum_{|r| \leqq m_{3}} c_{p q r} e^{2 \pi i(p x+q y+r z)} .
$$

From these sums we form the Fejér means

$$
\sigma_{m}(x, y, z)=\frac{1}{\left(m_{1}+1\right)\left(m_{2}+1\right)\left(m_{3}+1\right)} \sum_{k_{1}=0}^{m_{1}} \sum_{k_{2}=0}^{m_{2}} \sum_{k_{3}=0}^{m_{3}} s_{k_{1}, k_{2}, k_{3}}(x, y, z) .
$$


Since $0 \leqq \phi \leqq 1$, we have $0 \leqq \sigma_{\underline{m}} \leqq 1$. If $F$ is any closed subset of points of continuity in $C_{3}$, then $\sigma_{\underline{m}} \rightarrow \phi$ uniformly in $F$ as $|\underline{m}| \rightarrow \infty$ (c.f., A. Zygmund [8]). Let $W(p, q, r)$ be the limit computed in (4.10). Since each partial sum is a finite sum, we have

$$
\begin{aligned}
& \lim _{N \rightarrow \infty} \frac{1}{N} \sum_{n=1}^{N} s_{\underline{m}}\left(x_{n}, x_{n+1}, x_{n+2}\right) \\
&=\sum_{|p| \leqq m_{1}} \sum_{|q| \leqq m_{2}} \sum_{|r| \leqq m_{3}} c_{p q r} W_{(p, q, r)}=\frac{1}{6}+\sum_{r=1}^{t} \frac{\cos 4 \pi r \alpha}{2 \pi r^{2}}
\end{aligned}
$$

where $t$ is the largest integer for which $t \leqq m_{1}, 2 t \leqq m_{2}, t \leqq m_{3}$. We note that $t=t(\underline{m}) \rightarrow \infty$ as $|\underline{m}| \rightarrow \infty$. Let the limit (4.19) be called $s(\underline{m})$. As $|\underline{m}| \rightarrow \infty, s(\underline{m})$ tends to the limit

$$
s=\lim _{|\underline{m}| \rightarrow \infty} s(\underline{m})=\frac{1}{6}+\sum_{r=1}^{\infty} \frac{\cos 4 \pi r \alpha}{2 \pi r^{2}} .
$$

Replacing $s_{\underline{m}}$ by $\sigma_{\underline{m}}$ in $(4.19)$, we find

$$
\lim _{N \rightarrow \infty} \frac{1}{N} \sum_{n=1}^{N} \sigma_{\underline{m}}\left(x_{n}, x_{n+1}, x_{n+2}\right)=\sigma(\underline{m})
$$

where $\sigma(\underline{m})$ is the $(C, 1)$ mean value of the numbers $s(\underline{k})$ for $k_{1} \leqq m_{1}, k_{2} \leqq m_{2}$, $k_{3} \leqq m_{3}$. By the regularity of $(C, 1)$ summability, we have

$$
\sigma(\underline{m}) \rightarrow s=\frac{1}{6}+\sum_{r=1}^{\infty} \frac{\cos 4 \pi r \alpha}{2 \pi r^{2}} \text { as }|\underline{m}| \rightarrow \infty .
$$

Let $\epsilon>0$ be given. Define $F$ to be the closed set of points $(x, y, z)$ satisfying all the inequalities

$$
\begin{gathered}
\epsilon \leqq x \leqq 1-\epsilon, \quad \epsilon \leqq y \leqq 1-\epsilon, \quad \epsilon \leqq z \leqq 1-\epsilon, \\
|x-y| \geqq \epsilon, \quad|y-z| \geqq \epsilon .
\end{gathered}
$$

Since $\phi$ is continuous in $F$, there is a number $m_{0}=m_{0}(\epsilon)$ so large that

$$
\left|\phi(x, y, z)-\sigma_{\underline{m}}(x, y, z)\right|<\epsilon \text { in } F \text { if }|\underline{m}|>m_{0} .
$$

Let $R=C_{3}-F$. Let $P_{n}=\left(x_{n}, x_{n+1}, x_{n+2}\right)$. We have

$$
\begin{array}{r}
\sum_{n=1}^{N} \phi\left(P_{n}\right)-\sum_{n=1}^{N} \sigma_{\underline{m}}\left(P_{n}\right)=\sum_{\substack{n=1 \\
P_{n} \in \boldsymbol{F}}}^{N}\left(\phi\left(P_{n}\right)-\sigma_{\underline{m}}\left(P_{n}\right)\right)+\sum_{\substack{n=1 \\
P_{n} \in R}}^{N}\left(\phi\left(P_{n}\right)-\sigma_{\underline{m}}\left(P_{n}\right)\right) \\
=\sum_{1}+\sum_{2} .
\end{array}
$$

By (4.24) we have

$$
\left|\sum_{1}\right|<N \epsilon \quad \text { if } \quad|\underline{m}|>m_{0}(\epsilon) .
$$

Since $\phi$ and $\sigma_{\underline{m}}$ both lie between 0 and 1 , we have $\left|\sum_{2}\right| \leqq \nu_{N}=$ the number of points $P_{1}, \cdots, P_{N}$ which lie in $R$. By the definition (4.23) every point $P_{n}$ in $R$ satisfies at least one of the inequalities

$$
0 \leqq x_{n}<\epsilon, \quad 1-\epsilon<x_{n}<1, \quad 0 \leqq x_{n+1}<\epsilon, \quad 1-\epsilon<x_{n+1}<1,
$$

$$
0 \leqq x_{n+2}<\epsilon, \quad 1-\epsilon<x_{n+2}<1, \quad\left|x_{n}-x_{n+1}\right|<\epsilon, \quad\left|x_{n+1}-x_{n+2}\right|<\epsilon .
$$


But the sequence $x_{k}=\left\{k^{2} \alpha\right\}$ is equidistributed in $C_{1}$, and the sequence $\left(x_{k}, x_{k+1}\right)$ is equidistributed in $C_{2}$. Therefore,

$$
\begin{aligned}
\limsup _{N \rightarrow \infty} \frac{1}{N} \nu_{N} & \leqq \int_{0}^{\epsilon} d x+\int_{1-\epsilon}^{1} d x+\int_{0}^{\epsilon} d y+\int_{1-\epsilon}^{1} d y \\
+ & \int_{0}^{\epsilon} d z+\int_{1-\epsilon}^{1} d z+\iint_{|x-y|<\epsilon} d x d y+\iint_{|y-z|<\epsilon} d y d z<10 \epsilon .
\end{aligned}
$$

Letting $s$ be the limit (4.22), we find from (4.25)

$$
\left|\frac{1}{N} \sum_{n=1}^{N} \phi\left(P_{n}\right)-s\right| \leqq\left|\frac{1}{N} \sum_{n=1}^{N} \sigma_{\underline{m}}\left(P_{n}\right)-s\right|+\frac{1}{N}\left|\sum_{1}\right|+\frac{1}{N}\left|\sum_{2}\right|
$$

Let $N \rightarrow \infty$. If $|\underline{m}|>m_{0}(\epsilon)$ we find

$$
\limsup _{N \rightarrow \infty}\left|\frac{1}{N} \sum_{n=1}^{N} \phi\left(P_{n}\right)-s\right|<|\sigma(\underline{m})-s|+\epsilon+10 \epsilon .
$$

But by (4.22) $|\sigma(\underline{m})-s|<\epsilon$ if $|\underline{m}|$ is sufficiently large. Therefore, the limit superior $(4.30)$ is $<12 \epsilon$ for arbitrarily small $\epsilon>0$. Therefore,

$$
\lim _{N \rightarrow \infty} \frac{1}{N} \sum_{n=1}^{N} \phi\left(P_{n}\right)=s=\frac{1}{6}+\sum_{r=1}^{\infty} \frac{\cos 4 \pi r \alpha}{2 \pi r^{2}} .
$$

This justifies the required formula (4.12). The essential point in the proof was the inequality (4.28), which showed that not too many of the points $\left(x_{n}, x_{n+1}, x_{n+2}\right)$ fell near the discontinuities of $\phi$.

To discuss each of the other five orderings we proceed exactly as before. For example, to find those values of $\alpha$ which make $\operatorname{Pr}\left(x_{n}>x_{n+2}>x_{n+1}\right)=1 / 6$, we define the characteristic function $\phi(x, y, z)$ of the set $x>z>y$ in the unit cube $C_{3}$. This function has a Fourier series with coefficients $c_{p q r}$. Justifying the necessary interchange of limits, we find for this new function $\phi$ :

$$
\begin{aligned}
\operatorname{Pr}\left(x_{n}>x_{n+2}>x_{n+1}\right) & =\lim _{N \rightarrow \infty} \frac{1}{N} \sum_{n=1}^{N} \phi\left(x_{n}, x_{n+1}, x_{n+2}\right) \\
& =\sum_{r=-\infty}^{\infty} c_{r,-2 r, r} e^{4 \pi i r \alpha}=S(\alpha)
\end{aligned}
$$

where in this case

$$
\begin{aligned}
& c_{r}=c_{r,-2 r, r}=\int_{0}^{1} \int_{0}^{x} \int_{0}^{z} e^{-2 \pi i r(x-2 y+z)} d x d z d y \\
& c_{0}=1 / 6, \quad c_{r}=-\frac{1}{8 \pi^{2} r^{2}} \quad(r \neq 0) .
\end{aligned}
$$

This gives the sum

$$
\begin{array}{ll}
S(\alpha)=\frac{1}{8}+\frac{1}{2} \alpha-\alpha^{2} & \left(0 \leqq \alpha \leqq \frac{1}{2}\right) \\
S(\alpha)=S\left(\alpha-\frac{1}{2}\right) & \left(\frac{1}{2} \leqq \alpha \leqq 1\right) .
\end{array}
$$

This function $S(\alpha)$ is different from the function $S(\alpha)$ computed in (4.15) for the first ordering. Nevertheless, the new equation $S(\alpha)=1 / 6$ has the same roots as the old equation. 'These four roots in the interval $0<\alpha<1$ are, as before, 
$(3 \pm \sqrt{3}) / 12,(9 \pm \sqrt{3}) / 12 ;$ and $\operatorname{Pr}\left(x_{n}>x_{n+2}>x_{n+1}\right)=1 / 6$ if and only if $\alpha$ has one of these values.

For the remaining four cases these are the results: 'To compute the three probabilities $\operatorname{Pr}\left(x_{n+1}>x_{n}>x_{n+2}\right), \operatorname{Pr}\left(x_{n+1}>x_{n+2}>x_{n}\right), \operatorname{Pr}\left(x_{n+2}>x_{n}>x_{n+1}\right)$ we compute, respectively, the Fourier coefficients $c_{r}=c_{r,-2 r, r}$ for the characteristic functions $\phi$ of the sets $y>x>z, y>z>x, z>x>y$. For each of these cases computation gives the coefficients $c_{r}$ recorded in (4.33) for the second case. For the last case, to compute the probability $\operatorname{Pr}\left(x_{n+2}>x_{n+1}>x_{n}\right)$ we find for the characteristic function $\phi$ of the set $z>y>x$ the same coefficients $c_{r}$ which were computed in (4.13) for the first case. These unexpected coincidences show that the same four values of $\alpha$ make all of the six probabilities equal to $1 / 6$. This completes the proof of the theorem.

5. Completely Equidistributed Sequences. J. F. Koksma [3] proved in 1934 that for almost all $\theta>1$ the sequence $x_{n}=\left\{\theta^{n}\right\}$ is equidistributed. We shall show that these sequences, unlike the other equidistributed sequences which we have investigated, are completely equidistributed. We shall use the following preliminary result:

Theorem: (Koksma [3], Satz 3.) Let $\alpha$ and $\beta$ be fixed real numbers with $\alpha<\beta$. For each natural number $n$ let $f(n, \theta)$ be a real, continuously differentiable function of $\theta$ in $\alpha \leqq \theta \leqq \beta ;$ and let

$$
\frac{\partial}{\partial \theta} f(m, \theta)-\frac{\partial}{\partial \theta} f(n, \theta)
$$

denote for each pair of unequal natural numbers $m$ and $n$ a monotone function of $\theta$ in $\alpha \leqq \theta \leqq \beta$, which everywhere in this interval has absolute value $\geqq K$, where $K$ is positive and independent of $\theta, m$, and $n$. Then $f(n, \theta)(n=1,2, \cdots)$ is equidistributed modulo 1 for almost all $\theta$ in $\alpha \leqq \theta \leqq \beta$.

From this theorem we derive the following:

THEOREM 14. Let $p(\theta)$ be any twice continuously differentiable function with at most a finite number of zeros in any finite subinterval of $\theta>1$. For $n=1,2, \cdots$ let $M(n) \geqq 1$; and for each pair of positive integers $N \neq n$ let

$$
|M(N)-M(n)| \geqq L
$$

where $L$ is a positive number independent of $N$ and $n$. Then the function $p(\theta) \theta^{M(n)}$ $(n=1,2, \cdots)$ is for almost all $\theta>1$ equidistributed modulo 1.

For $p(\theta)=1$ this theorem was proved by Koksma [3], Satz 2 .

Proof. Let $1<\alpha<\beta$, where the closed interval $\alpha \leqq \theta \leqq \beta$ contains no zero of $p(\theta)$. The open interval between two consecutive zeros of $p(\theta)$ can be covered by a denumerable collection of closed intervals $[\alpha, \beta]$. Therefore, it will suffice to show that $p(\theta) \theta^{M(n)}$ is equidistributed modulo 1 for almost all $\theta$ in each interval $[\alpha, \beta]$. Let $g(\theta)=p(\theta) \theta^{M(n)}$. Let

$$
\psi(N, n, \theta)=\frac{\partial}{\partial \theta} g(N, \theta)-\frac{\partial}{\partial \theta} g(n, \theta) .
$$

We shall show that there is an integer $s \geqq 0$ such that, if $N>n>s$, then $\psi$ is a monotone function of $\theta$ in $[\alpha, \beta]$ with absolute value $\geqq K$, where $K$ is a positive 
number independent of $N, n$, and $\theta$. Then, by Koksma's theorem, the sequence $f(n, \theta)=g(n+s, \theta) \quad(n=1,2, \cdots)$ will have been shown to be equidistributed modulo 1 . But this will imply that the original sequence $g(n, \theta)(n=1,2 \cdots)$ is equidistributed modulo 1 , since the first $s$ members of a sequence cannot affect the property of equidistribution.

We show first that $M(n) \rightarrow \infty$ as $n \rightarrow \infty$. Given any $B>1$ we let $\nu \leqq \infty$ denote the number of integers $n$ such that $M(n) \leqq B$. Because of the inequality (5.2), we have $(\nu-1) L \leqq B-1$. Therefore, $\nu$ is finite. If $n_{B}$ is the greatest integer $n$ such that $M(n) \leqq B$, then $M(n)>B$ if $n>n_{B}$. Therefore, $M(n) \rightarrow \infty$ as $n \rightarrow \infty$.

Let $A$ and $a$ denote, respectively, the larger and the smaller of the values $M(N)$ and $M(n)$. Then

$$
\begin{aligned}
|\psi(N, n, \theta)| & =\left|\frac{\partial}{\partial \theta} \theta^{A} p(\theta)-\frac{\partial}{\partial \theta} \theta^{a} p(\theta)\right| \\
& =\left|p(\theta)\left(A \theta^{A-1}-a \theta^{a-1}\right)+p^{\prime}(\theta)\left(\theta^{A}-\theta^{a}\right)\right| .
\end{aligned}
$$

For $\alpha \leqq \theta \leqq \beta$ there are numbers $\epsilon>0$ and $D \geqq 0$ such that

$$
|p(\theta)| \geqq \epsilon, \quad\left|p^{\prime}(\theta)\right| \leqq D, \quad\left|p^{\prime \prime}(\theta)\right| \leqq D .
$$

Then

$$
\begin{aligned}
|\psi(N, n, \theta)| & \geqq \frac{\epsilon}{\theta}\left(A \theta^{A}-a \theta^{a}\right)-D\left(\theta^{A}-\theta^{a}\right) \\
& \geqq\left(\frac{\epsilon a}{\theta}-D\right) \theta^{a}\left(\theta^{A-a}-1\right) .
\end{aligned}
$$

But, by the assumption (5.2), $A-a \geqq L$. Therefore, for $\alpha \leqq \theta \leqq \beta$,

$$
|\psi(N, n, \theta)| \geqq K=\left(\frac{\epsilon a}{\beta}-D\right)\left(\alpha^{L}-1\right) .
$$

The number $K$ is positive if $a>D \beta / \epsilon$. Since $M(x) \rightarrow \infty$ as $x \rightarrow \infty$, there is a number $s$ so large that $M(x)>D \beta / \epsilon$ if $x>s$. Therefore,

$$
|\psi(N, n, \theta)| \geqq K>0 \text { if } N>n>s .
$$

It remains only to show that $\psi$ is monotone in $\theta$ for $N>n>$ sufficiently large $s$. We compute

$$
\begin{aligned}
&\left|\frac{\partial \psi(N, n, \theta)}{\partial \theta}\right|=\mid p(\theta)\left(A(A-1) \theta^{A-2}-a(a-1) \theta^{a-2}\right) \\
&+ 2 p^{\prime}(\theta)\left(A \theta^{A-1}-a \theta^{a-1}\right)+p^{\prime \prime}(\theta)\left(\theta^{A}-\theta^{a}\right) \mid \\
& \geqq \frac{\epsilon}{\theta^{2}}\left(A(A-1) \theta^{A}-a(a-1) \theta^{a}\right)-\frac{2 D}{\theta}\left(A \theta^{A}-a \theta^{a}\right)-D\left(\theta^{A}-\theta^{a}\right) \\
& \geqq \epsilon \theta^{-2} a\left((A-1) \theta^{A}-(a-1) \theta^{a}\right)-2 D \theta^{-1}\left(A \theta^{A}-a \theta^{a}\right)-D\left(\theta^{A}-\theta^{a}\right) \\
&=\left(\epsilon \theta^{-2} a-2 D \theta^{-1}\right)\left(A \theta^{A}-a \theta^{a}\right)-\left(D+\epsilon \theta^{-2} a\right)\left(\theta^{A}-\theta^{a}\right) .
\end{aligned}
$$

If $a>2 D \beta / \epsilon$, the last expression is

$$
\geqq\left(\epsilon \theta^{-2} a^{2}-\left(2 D \theta^{-1}+\epsilon \theta^{-2}\right) a-D\right)\left(\theta^{A}-\theta^{a}\right) .
$$


The last expression is positive for all $\theta$ in $[\alpha, \beta]$ if

$$
\epsilon \beta^{-2} a^{2}-\left(2 D \alpha^{-1}+\epsilon \alpha^{-2}\right) a-D>0
$$

which is true for all sufficiently large $a$. Therefore, if $s$ is sufficiently large,

$$
\frac{\partial \psi}{\partial \theta}(N, n, \theta) \neq 0 \quad \text { if } \quad N>n>s .
$$

This gives the required monotonicity; inequalities (5.8) and (5.9) complete the proof of the theorem.

Theorem 15. For any two functions $A(\theta), B(\theta)$ the sequence $x_{n}=\left\{A(\theta) \theta^{n}+\right.$ $B(\theta)\}(n=1,2, \cdots)$ is completely equidistributed for almost all $\theta>1$ if $A(\theta)$ has two continuous derivatives for $\theta>1$ and if $A(\theta)$ has at most a finite number of zeros in any finite subinterval of $\theta>1$.

Proof. For every integer $r>0$ we must show that for almost all $\theta>1$ the sequence of vectors $z^{(n)}=\left(x_{n}, x_{n+1}, \cdots, x_{n+r-1}\right)(n=1,2, \cdots)$ is equidistributed in the $r$-dimensional unit cube $C_{r}$. By the Weyl criterion this is equivalent to showing that

$$
\frac{1}{N} \sum_{n=1}^{N} \exp 2 \pi i \sum_{\rho=0}^{r-1} k_{\rho}\left(A(\theta) \theta^{n+\rho}+B(\theta)\right) \rightarrow 0 \text { as } N \rightarrow \infty
$$

for almost all $\theta>1$ if $k_{0}, \cdots, k_{r-1}$ are any integers not all zero. Let $q(\theta)=k_{0}+$ $k_{1} \theta+\cdots+k_{r-1} \theta^{r-1}$. Formula (5.10) requires that

$$
\frac{1}{N} \sum_{n=1}^{N} \exp 2 \pi i q(\theta) A(\theta) \theta^{n} \rightarrow 0 \quad \text { as } \quad N \rightarrow \infty .
$$

But the function $p(\theta)=q(\theta) A(\theta)$ satisfies the conditions of the preceding theorem, since the polynomial $q$ has at least one non-zero coefficient. Therefore, for almost all $\theta>1$ the sequence $\left\{q(\theta) A(\theta) \theta^{n}\right\}$ is equidistributed in $C_{1}$. Then (5.11) follows from the one-dimensional form of the Weyl criterion applied to the sequence $q(\theta) A(\theta) \theta^{n}$. This completes the proof.

Corollary. The sequence $\left\{\theta^{n}\right\}(n=1,2, \cdots)$ is completely equidistributed for almost all $\theta>1$.

Proof. In the last theorem this is the case $A(\theta)=1, B(\theta)=0$.

The algebraic character of the number $\theta$ may influence the sequential properties of $\left\{\theta^{n}\right\}$. Although it is not known whether $\left\{\theta^{n}\right\}$ may be equidistributed if $\theta$ is rational, we have the result:

ThEOREM 16. If $\left\{\theta^{n}\right\}$ is equidistributed by $r$ 's, then $\theta$ cannot be an algebraic number of degree $<r$. In particular, if $\left\{\theta^{n}\right\}$ is completely equidistributed, then $\theta$ is transcendental.

Proof. By (5.11), if $\left\{\theta^{n}\right\}$ is equidistributed by $r$ 's, then

$$
\frac{1}{N} \sum_{n=1}^{N} \exp 2 \pi i\left(k_{0}+k_{1} \theta+\cdots+k_{r-1} \theta^{r-1}\right) \theta^{n} \rightarrow 0 \quad \text { as } \quad N \rightarrow \infty
$$

if $k_{0}, \cdots, k_{r-1}$ are distinct integers not all zero. Therefore, $q(\theta) \neq 0$ for any polynomial $q$ of degree $<r$ with integer coefficients not all zero. 
Theonem 17. Every completely equidistributed sequence $x_{n}$ is white.

Proof. For every $\tau=1,2, \cdots$ the pairs $\left(x_{n}, x_{n+\tau}\right)$ are equidistributed in $C_{2}$, since for $0 \leqq a_{0}<b_{0} \leqq 1,0 \leqq a_{\tau}<b_{\tau} \leqq 1$

$$
\begin{array}{r}
\operatorname{Pr}\left(a_{0} \leqq x_{n}<b_{0}, \quad a_{\tau} \leqq x_{n+\tau}<b_{\tau}\right) \\
=\operatorname{Pr}\left(a_{0} \leqq x_{n}<b_{0}, \quad 0 \leqq x_{n+1}<1, \cdots, \quad 0 \leqq x_{n+\tau-1}<1, \quad a_{\tau} \leqq x_{n+\tau}<b_{\tau}\right) \\
=\left(b_{0}-a_{0}\right)\left(b_{\tau}-a_{\tau}\right)
\end{array}
$$

because the sequence $x_{n}$ is equidistributed by $(\tau+1)$ 's. Therefore, as in the proof of Theorem 12, $x_{n}$ is white.

6. White Sequences. We have called an equidistributed sequence $x_{n}$ white if it is uncorrelated with any of its translates $x_{n+\tau}, \tau \neq 0$ :

$$
R(\tau)=\lim _{N \rightarrow \infty} \frac{1}{N} \sum_{n=1}^{N}\left(x_{n}-\frac{1}{2}\right)\left(x_{n+\tau}-\frac{1}{2}\right)=0 \quad(\tau=1,2, \cdots) .
$$

We have shown in the proof of Theorem 12 that every sequence $x_{n}$ is white for which the pairs $\left(x_{n}, x_{n+\tau}\right)$ are equidistributed in $C_{2}$ for every $\tau=1,2, \cdots$. The purpose of this section is to emphasize that whiteness is a weak criterion of randomness. We shall show that an equidistributed white sequence need not be equipartitioned by twos.

Theorem 18. There is an equidistributed white sequence $x_{n}$ for which $\operatorname{Pr}\left(x_{n}>x_{n+1}\right)$ $>\frac{1}{2}$.

Proof. Let $y_{1}, y_{2}, \cdots$ be any truly random sequence of independent samples from the uniform distribution on $0 \leqq y<1$. We shall form the sequence $x_{1}, x_{2}, \cdots$ from the separate pairs $y_{1}, y_{2} ; y_{3}, y_{4} ; \cdots$ of the $y$-sequence. Let $G$ be a fixed region in the unit square $0 \leqq u<1,0 \leqq v<1$; let $G^{*}$ be the complementary set. We define

$$
\begin{array}{lll}
x_{2 n-1}=y_{2 n-1}, x_{2 n}=y_{2 n} & \text { if } & \left(y_{2 n-1}, y_{2 n}\right) \in G \\
x_{2 n-1}=y_{2 n}, x_{2 n}=y_{2 n-1} & \text { if } & \left(y_{2 n-1}, y_{2 n}\right) \in G^{*}
\end{array}
$$

For any $G$ this transformation leaves the sequence $x_{n}$ equidistributed, since for every $N=1,2, \cdots$

$$
\left|\sum_{\substack{a \leqq n_{n}<b \\ n \leqq N}} 1-\sum_{\substack{a \leqq y_{n}<b \\ n \leqq N}} 1\right| \leqq 1
$$

For any $G$ we shall compute the autocorrelation function $R(\tau)$ of the $x$-sequence and compute $\operatorname{Pr}\left(x_{n}>x_{n+1}\right)$. We shall then choose $G$ to make $R(\tau)=0$ for $\tau \neq 0$ but make $\operatorname{Pr}\left(x_{n}>x_{n+1}\right)>\frac{1}{2}$.

Let $g$ be the area of $G$; we assume $0<g<1$. Let $\alpha$ be the area of the intersection of $G$ with the triangle $0 \leqq v<u<1$, and let $\beta$ be the area of the intersection of $G$ with the triangle $0 \leqq u<v<1$; thus $\alpha+\beta=g$. Let $\gamma$ and $\delta$ be the moments

$$
\gamma=\frac{1}{g} \iint_{G}\left(u-\frac{1}{2}\right) d u d v, \quad \delta=\frac{1}{g} \iint_{\sigma}\left(v-\frac{1}{2}\right) d u d v .
$$


Let numbers $g^{*}, \alpha^{*}, \beta^{*}, \gamma^{*}, \delta^{*}$ be defined analogously with respect to $G^{*}$. Thus $\alpha^{*}+\dot{\beta}^{*}=g^{*}, \alpha+\alpha^{*}=1 / 2, \beta+\beta^{*}=1 / 2, g+g^{*}=1$, and

$$
g\left(\begin{array}{l}
\gamma \\
\delta
\end{array}\right)+g^{*}\left(\begin{array}{l}
\gamma^{*} \\
\delta^{*}
\end{array}\right)=\left(\begin{array}{l}
0 \\
0
\end{array}\right) .
$$

Let $P_{n}=\left(y_{2 n-1}, y_{2 n}\right)(n=1,2, \cdots)$. Finally, let $a_{n}=x_{n}-\frac{1}{2}, b_{n}=y_{n}-\frac{1}{2}$.

First we compute $R(1)=E a_{n} a_{n+1}$. If $n$ is odd, $a_{n}$ and $a_{n+1}$ come from the same point $P_{n}$. Therefore,

$$
E a_{2 n-1} a_{2 n}=E b_{2 n-1} b_{2 n}=0
$$

since the numbers $b_{n}=y_{n}-1 / 2$ are uncorrelated with mean zero. If $n$ is even, the numbers $a_{n}, a_{n+1}$ come from two consecutive points $P$. Thus

$$
\begin{array}{rlll}
\left(a_{2 n}, a_{2 n+1}\right)=\left(b_{2 n}, b_{2 n+1}\right) & \text { if } & P_{n} \in G, P_{n+1} \in G \\
=\left(b_{2 n}, b_{2 n+2}\right) & \text { if } & P_{n} \in G, P_{n+1} \in G^{*} \\
=\left(b_{2 n-1}, b_{2 n+1}\right) & \text { if } & P_{n} \in G^{*}, P_{n+1} \in G \\
=\left(b_{2 n-1}, b_{2 n+2}\right) & \text { if } & P_{n} \in G^{*}, P_{n+1} \in G^{*} .
\end{array}
$$

Therefore, as we shall explain directly,

$$
E a_{2 n} a_{2 n+1}=g^{2} \delta \gamma+g g^{*} \delta \delta^{*}+g^{*} g \gamma^{*} \gamma+g^{* 2} \gamma^{*} \delta^{*} .
$$

The first term $g^{2} \delta \gamma$ is simply the probability $g^{2}$ that both points $P_{n}, P_{n+1}$ lie in $G$, multiplied by the mean values $\delta, \gamma$ of $b_{2 n}=v-\frac{1}{2}$ in $G$ and of $b_{2 n+1}=u-\frac{1}{2}$ in $G$; the other three terms arise similarly. Using the identities $g^{*} \gamma^{*}=-g \gamma, g^{*} \delta^{*}=-g \delta$, we find from (6.7)

$$
E a_{2 n} a_{2 n+1}=-g^{2}(\gamma-\delta)^{2}
$$

Since

$$
R(1)=E a_{n} a_{n+1}=\frac{1}{2}\left(E a_{2 n-1} a_{2 n}+E a_{2 n} a_{2 n+1}\right)
$$

we have from (6.5) and (6.8)

$$
R(1)=-\frac{1}{2} g^{2}(\gamma-\delta)^{2} .
$$

Next we compute $R(\tau)$ for $\tau=2 s=2,4,6, \cdots$. We have

$$
R(2 s)=E\left(a_{n} a_{n+2 s}\right)=\frac{1}{2} E\left(a_{2 n-1} a_{2 n-1+2 s}+a_{2 n} a_{2 n+2 s}\right) .
$$

But

$a_{2 n-1} a_{2 n-1+2 s}+a_{2 n} a_{2 n+2 s}$

$$
\begin{aligned}
& =b_{2 n-1} b_{2 n-1+2 s}+b_{2 n} b_{2 n+2 s} \quad \text { if } \quad P_{n} \in G, P_{n+s} \in G \\
& =b_{2 n-1} b_{2 n+2 s}+b_{2 n} b_{2 n-1+28} \quad \text { if } \quad P_{n} \in G, P_{n+s} \in G^{*} \\
& \text { (6.11) }=b_{2 n} b_{2 n-1+2 s}+b_{2 n-1} b_{2 n+2 s} \quad \text { if } P_{n} \in G^{*}, P_{n+s} \in G \\
& =b_{2 n} b_{2 n+2 s}+b_{2 n-1} b_{2 n-1+2 s} \quad \text { if } \quad P_{n} \in G^{*}, P_{n+1} \in G^{*} \text {. }
\end{aligned}
$$

Therefore, $E\left(a_{2 n-1} a_{2 n-1+2 s}+a_{2 n} a_{2 n+2 s}\right)$ equals $g^{2}\left(\gamma^{2}+\delta^{2}\right)+g g^{*}\left(\gamma \delta^{*}+\delta \gamma^{*}\right)+g^{*} g\left(\delta^{*} \gamma+\gamma \delta^{*}\right)+g^{* 2}\left(\delta^{* 2}+\gamma^{* 2}\right)=2 g^{2}(\gamma-\delta)^{2}$. 
Therefore,

$$
R(2 s)=g^{2}(\gamma-\delta)^{2} \quad(s=1,2, \cdots) .
$$

The cases $\tau=2 s+1 \quad(s=1,2, \cdots)$ are slightly more complicated. We have

$$
\begin{aligned}
a_{2 n-1} a_{2 n+2 s} & =b_{2 n-1} b_{2 n+2 s} & \text { if } & P_{n} \in G, P_{n+s} \in G \\
& =b_{2 n-1} b_{2 n+2 s-1} & \text { if } & P_{n} \in G, P_{n+s} \in G^{*} \\
& =b_{2 n} b_{2 n+2 s} & \text { if } & P_{n} \in G^{*}, P_{n+s} \in G \\
& =b_{2 n} b_{2 n+2 s-1} & \text { if } & P_{n} \in G^{*}, P_{n+s} \in G^{*} .
\end{aligned}
$$

But a term $a_{2 n} a_{2 n+2 s+1}$ will involve a third point, $P_{n+s+1}$ :

$$
\begin{aligned}
& a_{2 n} a_{2 n+2 s+1}=b_{2 n} b_{2 n+2 s+1} \quad \text { if } \quad P_{n} \in G, P_{n+s+1} \in G \\
& =b_{2 n} b_{2 n+2 s+2} \quad \text { if } \quad P_{n} \in G, P_{n+s+1} \in G^{*} \\
& =b_{2 n-1} b_{2 n+2 s+1} \quad \text { if } \quad P_{n} \in G^{*}, P_{n+s+1} \in G \\
& =b_{2 n-1} b_{2 n+28+2} \quad \text { if } \quad P_{n} \in G^{*}, P_{n+s+1} \in G^{*} \text {. }
\end{aligned}
$$

Therefore, for $s=1,2, \cdots$,

$2 R(2 s+1)=E a_{2 n-1} a_{2 n+2 s}+E a_{2 n} a_{2 n+2 s+1}=g^{2} \gamma \delta+g g^{*} \gamma \gamma^{*}+g^{*} g \delta^{*} \delta+g^{* 2} \delta^{*} \gamma^{*}$

$$
+g^{2} \delta \gamma+g g^{*} \delta \delta^{*}+g^{*} g \gamma^{*} \gamma+g^{* 2} \gamma^{*} \delta^{*}=2 g^{2}\left(\gamma \delta-\gamma^{2}-\delta^{2}+\delta \gamma\right)
$$

$$
R(2 s+1)=-g^{2}(\gamma-\delta)^{2} \quad(s=1,2, \cdots) .
$$

Having computed $R(\tau)$ for all $\tau \neq 0$, we shall compute $\operatorname{Pr}\left(x_{n}>x_{n+1}\right)$. If $n$ is odd, the numbers $y_{n}, y_{n+1}$ are the coordinates $u, v$ of the same point $P_{j}$, where $n=2 j-1$. But

$$
\begin{aligned}
\left(x_{2 j-1}, x_{2 j}\right)=\left(y_{2 j-1}, y_{2 j}\right) & \text { if } \quad P_{j} \in G \\
& =\left(y_{2 j}, y_{2 j-1}\right) \quad \text { if } \quad P_{j} \in G^{*} .
\end{aligned}
$$

Therefore, $x_{2 j-1}>x_{2 j}$ when $(u, v)=P_{j} \in G$ and $u>v$, or $P_{j} \in G^{*}$ and $v>u$. By the definitions of the areas $\alpha, \beta, \alpha^{*}, \beta^{*}$

$$
\operatorname{Pr}\left(x_{2 j-1}>x_{2 j}\right)=\alpha+\beta^{*}=\alpha+\frac{1}{2}-\beta .
$$

When $n=2 j$ is even, the numbers $x_{2 j}, x_{2 j+1}$ come from different points $P_{j}, P_{j+1}$. We have

$$
\operatorname{Pr}\left(x_{2 j}>x_{2 j+1}\right)=g^{2} A+g g^{*} B+g^{*} g C+g^{* 2} D
$$

where, if $P_{j}=\left(u_{j}, v_{j}\right)=\left(y_{2 j-1}, y_{2 j}\right)$,

$$
\begin{aligned}
& A=\operatorname{Pr}\left(v_{j}>u_{j+1} \mid P_{j} \in G, P_{j+1} \in G\right) \\
& B=\operatorname{Pr}\left(v_{j}>v_{j+1} \mid P_{j} \in G, P_{j+1} \in G^{*}\right) \\
& C=\operatorname{Pr}\left(u_{j}>u_{j+1} \mid P_{j} \in G^{*}, P_{j+1} \in G\right) \\
& D=\operatorname{Pr}\left(u_{j}>v_{j+1} \mid P_{j} \in G^{*}, P_{j+1} \in G^{*}\right) .
\end{aligned}
$$


We must now define four probability densities $\phi(u), \psi(v), \phi^{*}(u), \psi^{*}(v)$. We define

$$
\begin{aligned}
\phi(u) d u & =\operatorname{Pr}\left(u \leqq u^{\prime} \leqq u+d u \mid\left(u^{\prime}, v^{\prime}\right) \in G\right) \\
\psi(v) d v & =\operatorname{Pr}\left(v \leqq v^{\prime} \leqq v+d v \mid\left(u^{\prime}, v^{\prime}\right) \in G\right)
\end{aligned}
$$

or, equivalently,

$$
\begin{aligned}
& \Phi(u)=\int_{0}^{u} \phi\left(u^{\prime}\right) d u^{\prime}=\frac{1}{g} \iint_{\substack{\left(u^{\prime}, v^{\prime}\right) \in G \\
u^{\prime} \leqq u}} d u^{\prime} d v^{\prime} \\
& \Psi(v)=\int_{0}^{v} \psi\left(v^{\prime}\right) d v^{\prime}=\frac{1}{g} \iint_{\substack{\left(u^{\prime}, v^{\prime}\right) \in G \\
v^{\prime} \leqq v}} d u^{\prime} d v^{\prime} .
\end{aligned}
$$

The probability densities $\phi^{*}(u), \psi^{*}(v)$ and their integrals $\Phi^{*}, \Psi^{*}$ are defined analogously for the region $G^{*}$. Thus

$$
g \phi(u)+g^{*} \phi^{*}(u)=1, \quad g \psi(v)+g^{*} \psi^{*}(v)=1 .
$$

From (6.16) we now compute

$$
\begin{aligned}
& A=\int_{0}^{1} \int_{0}^{v_{j}} \psi\left(v_{j}\right) \phi\left(u_{j+1}\right) d u_{j+1} d v_{j} \\
& A=\int_{0}^{1} \psi(v) \Phi(v) d v .
\end{aligned}
$$

Similarly we compute

$$
\begin{aligned}
& B=\int_{0}^{1} \psi(v) \Psi^{*}(v) d v \\
& C=\int_{0}^{1} \phi^{*}(u) \Phi(u) d u \\
& D=\int_{0}^{1} \phi^{*}(u) \Psi^{*}(u) d u .
\end{aligned}
$$

From (6.15) we now find $\operatorname{Pr}\left(x_{2 j}>x_{2 j+1}\right)$ equal to

$$
\int_{0}^{1}\left[g^{2} \psi(t) \Phi(t)+g g^{*} \psi \Psi^{*}+g^{*} g \phi^{*} \Phi+g^{* 2} \phi^{*} \Psi^{*}\right] d t .
$$

From (6.19) we see that the integrand equals

$$
\begin{aligned}
g^{2} \psi \Phi+g \psi(t-g \Psi) & +g \Phi(1-g \phi)+(1-g \phi)(t-g \Psi) \\
& =g^{2} \frac{d}{d t}\left(\Psi \Phi-\frac{1}{2}\left(\Psi^{2}+\Phi^{2}\right)\right)+g \frac{d}{d t}(t \Phi-t \Psi)+t+2 g t(\psi-\phi) .
\end{aligned}
$$


Integration gives

$$
\begin{aligned}
\operatorname{Pr}\left(x_{2 j}>x_{2 j+1}\right) & =\int_{0}^{1}(t+2 g t(\psi-\phi)) d t \\
& =\frac{1}{2}+2 g E(v-u \mid(u, v) \in G) \\
\operatorname{Pr}\left(x_{2 j}>x_{2 j+1}\right) & =\frac{1}{2}+2 g(\delta-\gamma) .
\end{aligned}
$$

From (6.14) and (6.23) we conclude

$$
\operatorname{Pr}\left(x_{n}>x_{n+1}\right)=\frac{1}{2}(1+\alpha-\beta)+g(\delta-\gamma) .
$$

Now we are ready to define $G$. We have shown for $s=1,2, \cdots$

$$
\begin{aligned}
R(1)=-\frac{1}{2} g^{2}(\gamma-\delta)^{2}, \quad R(2 s)=g^{2}(\gamma-\delta)^{2}, \\
R(2 s+1)=-g^{2}(\gamma-\delta)^{2} .
\end{aligned}
$$

Therefore, it will suffice to pick $G$ to be any region which has its centroid $\left(\gamma+\frac{1}{2}, \delta+\frac{1}{2}\right)$ on the line $u=v$, but which has more area $\alpha$ to the right of $u=v$ than it has area $\beta$ to the left of $u=v$. This we may do, for example, by letting $G$ consist of two small circles $K$ and $k$, where $K$ has area $2 \epsilon>0$ and lies to the right of $u=v$, and where $k$ has area $\epsilon$ and lies to the left of $u=v$. Let $K$ have center $O$ and $k$ have center $o$. We require that the line segment connecting $O$ to $o$ pass through the center of the square $\left(\frac{1}{2}, \frac{1}{2}\right)$, and that the distance from the center of the square to $o$ be twice the distance to $O$. Then $\gamma=\delta, \alpha=2 \epsilon, \beta=\epsilon$,

$$
\operatorname{Pr}\left(x_{n}>x_{n+1}\right)=\frac{1}{2}(1+\epsilon), \quad R(\tau)=0 \quad(\tau=1,2, \cdots) .
$$

For example, if $0<\epsilon<\pi / 128$, we may let $K$ and $k$ be the circles

$$
\left(u-\frac{5}{8}\right)^{2}+\left(v-\frac{3}{8}\right)^{2}<\frac{2 \epsilon}{\pi}, \quad\left(u-\frac{1}{4}\right)^{2}+\left(v-\frac{3}{4}\right)^{2}<\frac{\epsilon}{\pi} .
$$

This completes the proof of the theorem.

7. Sequential Equidistribution in Higher Dimensions. We have so far considered various sequential properties of sequences $x_{1}, x_{2}, \cdots$ equidistributed in the one-dimensional line segment $C_{1}$. But for many applications we must simulate random sequences in higher-dimensional cubes $C_{r}$; typically $r=2$ or 3 . For these applications we require sequences of $r$-dimensional vectors

$$
y^{(n)}=\left(y_{1}{ }^{(n)}, y_{2}{ }^{(n)}, \cdots, y_{r}{ }^{(n)}\right) \quad(n=1,2, \cdots)
$$

which are equidistributed in $C_{r}$. One can discuss various extensions of the notion of equipartition. It might also be useful to discuss the autocorrelation function

$$
R(\tau)=\lim _{N \rightarrow \infty} \frac{1}{N} \sum_{n=1}^{N} \sum_{\rho=1}^{r}\left(y_{\rho}^{(n)}-\frac{1}{2}\right)\left(y_{\rho}^{(n+\tau)}-\frac{1}{2}\right) \quad(\tau=0,1, \cdots)
$$

to define the related spectral density $\phi(\omega)$, to extend Jagerman's results to higher dimensions, and to discuss higher-dimensional white sequences.

However, in this paper we shall consider extensions only of the notions of 
equidistribution by $k$ 's and of complete equidistribution. For any $k=1,2, \cdots$ we shall say that the sequence of $r$-dimensional vectors $y^{(n)}$ is equidistributed by $k$ 's if the sequence of $k \cdot r$ dimensional vectors

$$
\begin{aligned}
w^{(n)} & =\left(y^{(n)}, \cdots, y^{(n+k-1)}\right) \\
& =\left(y_{1}{ }^{(n)}, \cdots, y_{r}{ }^{(n)}, y_{1}{ }^{(n+1)}, \cdots, y_{r}{ }^{(n+1)}, \cdots, y_{1}{ }^{(n+k-1)}, \cdots, y_{r}{ }^{(n+k-1)}\right)
\end{aligned}
$$

is equidistributed in the $k \cdot r$ dimensional unit cube $C_{k r}$. The sequence $y^{(n)}$ is completely equidistributed if it is equidistributed by $k$ 's for all $k$.

If $x_{1}, x_{2}, \cdots$ is a truly random sequence of independent samples from the uniform distribution on $C_{1}$, then

$$
y^{(n)}=\left(x_{n r}, x_{n r+1}, \cdots, x_{n r+r-1}\right) \quad(n=1,2, \cdots)
$$

provides a truly random sequence of independent samples from the uniform distribution on $C_{r}$.

For any one-dimensional sequence $x_{n}$, random or deterministic, we define the $r$-dimensional "derived sequence" $z^{(n)}$ by (7.4). We shall investigate the derived sequences of certain one-dimensional equidistributed sequences.

We first ask whether a sequence $x_{n}$ equidistributed by $r$ 's for some $r>1$ has an equidistributed $r$-dimensional derived sequence. This is not true in general.

Theorem 19. There is a sequence $x_{1}, x_{2}, x_{3}, \cdots$ equidistributed by 2's in $C_{1}$ for which the 2-dimensional derived sequence

$$
y^{(1)}=\left(x_{2}, x_{3}\right), \quad y^{(2)}=\left(x_{4}, x_{5}\right), \quad y^{(3)}=\left(x_{6}, x_{7}\right), \cdots
$$

is not equidistributed in $C_{2}$.

Proof. We shall construct a sequence $x_{n}$ for which the pairs $\left(x_{n}, x_{n+1}\right)$ are equidistributed in $C_{2}$ but for which the alternate pairs $\left(x_{2 j}, x_{2 j+1}\right)$ are not equidistributed. Let $g_{n}$ be any sequence equidistributed by twos, e.g., $g_{n}=\left\{n^{2} \alpha\right\}, \alpha$ irrational. If we let I and II represent, respectively, the left and the right halves of the interval $0 \leqq x<1$, we create from the $g$-sequence a sequence $x_{n}$ which may be represented schematically as follows:

$$
x_{n}=\mathrm{I}, \mathrm{I}, \mathrm{II}, \mathrm{II}, \mathrm{I}, \mathrm{I}, \mathrm{II}, \mathrm{II}, \cdots .
$$

To be precise, we define

$$
\begin{array}{llll}
x_{1}=\frac{1}{2} g_{1}, & x_{2}=\frac{1}{2} g_{2}, & x_{3}=\frac{1}{2}+\frac{1}{2} g_{3}, & x_{4}=\frac{1}{2}+\frac{1}{2} g_{4}, \\
x_{5}=\frac{1}{2} g_{5}, & x_{6}=\frac{1}{2} g_{6}, & x_{7}=\frac{1}{2}+\frac{1}{2} g_{7}, & x_{8}=\frac{1}{2}+\frac{1}{2} g_{8}, \text { etc. }
\end{array}
$$

The successive pairs $\left(x_{n}, x_{n+1}\right)$ have the schematic representation

$$
\left(x_{n}, x_{n+1}\right)=(\mathrm{I}, \mathrm{I}),(\mathrm{I}, \mathrm{II}),(\mathrm{II}, \mathrm{II}),(\mathrm{II}, \mathrm{I}), \cdots \text {. }
$$

Thus the pairs $\left(x_{n}, x_{n+1}\right)$ are equidistributed in the four sub-squares of $C_{2}$ :

$$
\begin{array}{ll}
(\mathrm{I}, \mathrm{II})=\left(0 \leqq u<\frac{1}{2}, \frac{1}{2} \leqq v<1\right) & (\mathrm{II}, \mathrm{II})=\left(\frac{1}{2} \leqq u<1, \frac{1}{2} \leqq v<1\right) \\
(\mathrm{I}, \mathrm{I})=\left(0 \leqq u<\frac{1}{2}, 0 \leqq v<\frac{1}{2}\right) & (\mathrm{II}, \mathrm{I}) \leqq\left(\frac{1}{2} \leqq u<1,0 \leqq v<\frac{1}{2}\right) .
\end{array}
$$

Therefore, $\left(x_{n}, x_{n+1}\right)$ is equidistributed in $C_{2}$. But the successive pairs $\left(x_{2 j}, x_{2 j+1}\right)$ have the schematic representation

$$
\left(x_{2 j}, x_{2 j+1}\right)=(\mathrm{I}, \mathrm{II}),(\mathrm{II}, \mathrm{I}),(\mathrm{I}, \mathrm{II}),(\mathrm{II}, \mathrm{I}), \cdots .
$$


Since the sub-squares (I, I) and (II, II) remain empty, the sequence $\left(x_{2 j}, x_{2 j+1}\right)$ cannot be equidistributed in $C_{2}$. This completes the proof.

Theorem 20. For some $x_{0}$ let $x_{n+1}=\left\{N x_{n}+\theta\right\} \quad(n=0,1, \cdots), N=$ integer $\geqq 2$. For any such sequence the $r$-dimensional derived sequence (7.4) cannot be equidistributed in $C_{r}$ for any $r>1$.

Proof. Suppose that for some $r>1$ the derived sequence $y^{(n)}$ defined by (7.4) were equidistributed. From this assumption we shall deduce that the original sequence $x_{n}$ is equidistributed by $r$ 's. Let $h$ be the $r$-dimensional vector $h=(\theta, \theta, \cdots, \theta)$. Since $N$ is an integer $\neq 0$, it is an immediate consequence of the Weyl criterion for equidistribution in $C_{r}$ that the sequence

$$
y^{(n, 1)}=N y^{(n)}+h \quad(n=1,2, \cdots)
$$

is also equidistributed modulo 1 in $C_{r}$. Consequently,

$$
y^{(n, 2)}=N y^{(n, 1)}+h \quad(n=1,2, \cdots)
$$

is equidistributed modulo 1 in $C_{r}$, et cetera; each of the $r$ sequences $y^{(n)}, y^{(n, 1)}, \cdots$, $y^{(n, r-1)}$ is equidistributed modulo 1 in $C_{r}$. But, since $N x_{k}+\theta \equiv x_{k+1}$ modulo 1 ,

$$
\begin{array}{rlr}
y^{(n)} & =\left(x_{n r}, x_{n r+1}, \cdots, x_{n r+r-1}\right) & \\
y^{(n, 1)} & \equiv\left(x_{n r+1}, x_{n r+2}, \cdots, x_{n r+r}\right) & (\bmod 1) \\
\cdot & \cdot & \cdots \\
y^{(n, r-1)} & \equiv\left(x_{n r+r-1}, x_{n r+r}, \cdots, x_{n r+2 r-2}\right) & (\bmod 1) .
\end{array}
$$

To show that the original sequence $x_{n}$ is equidistributed by $r$ 's we must show that the sequence of vectors $z^{(k)}=\left(x_{k}, x_{k+1}, \cdots x_{k+r-1}\right)(k=1,2, \cdots)$ is equidistributed in $C_{r}$. But

$$
z^{(r)}, z^{(r+1)}, \cdots \equiv y^{(1)}, y^{(1,1)}, \cdots, y^{(1, r-1)}, y^{(2)}, y^{(2,1)}, \cdots, y^{(2, r-1)}, \cdots .
$$

In general, for $k \geqq r$, if $y^{(n, 0)}=y^{(n)}$,

$$
z^{(k)} \equiv y^{(n, \rho)} \quad(\rho=0, \cdots, r-1 ; n=1,2, \cdots ; k=n r+\rho) .
$$

Therefore, $z^{(k)}$ is equidistributed in $C_{r}$, and the Multiply sequence $x_{n}$ is equidistributed by $r$ 's. But this is a contradiction to Theorem 4, which implies that no Multiply sequence is equidistributed by $r$ 's for any $r>1$.

Theorem 21. Let $\alpha$ be irrational. Let

$$
x_{n}=\left\{\alpha n^{p}+c_{1} n^{p-1}+c_{2} n^{p-2}+\cdots+c_{p}\right\} \quad(n=1,2, \cdots) .
$$

The $r$-dimensional derived sequence $y^{(n)}$ is equidistributed by $k$ 's if and only if $k r \leqq p$.

Proof. For $r=1$ this theorem reduces to Theorem 11 in Section 4. The sequence of $y^{(n)}$ is equidistributed by $k$ 's when the sequence

$$
w^{(n)}=\left(x_{n r}, x_{n r+1}, \cdots, x_{n r+k r-1}\right) \quad(n=1,2, \cdots)
$$

is equidistributed in $C_{k r}$. We now proceed as in the proof of Theorem 11. Let $f(n)=\alpha n^{p}+\cdots+c_{p}$. Let $\Delta f(m)=f(m+1)-f(m)$. The Weyl criterion states that $w^{(n)}$ is equidistributed when

$$
\lim _{N \rightarrow \infty} N^{-1} \sum_{n=1}^{N} \exp 2 \pi i \sum_{j=0}^{k r-1} h_{j} f(n r+j)=0
$$


for any integers $h_{0}, \cdots, h_{k r-1}$ not all zero, or, equivalently, when

$$
\lim _{N \rightarrow \infty} N^{-1} \sum_{n=1}^{N} \exp 2 \pi i \sum_{j=0}^{k r-1} h_{j}^{\prime} \Delta^{j} f(n r)=0
$$

for any integers $h_{0}{ }^{\prime}, \cdots, h_{k r-1}^{\prime}$ not all zero.

If $k r>p$, we may choose $h_{p}{ }^{\prime}=1, h_{j}{ }^{\prime}=0 \quad(j \neq p)$. Then for every $N=$ $1,2, \cdots$

$$
\begin{aligned}
N^{-1} \sum_{n=1}^{N} \exp 2 \pi i \sum_{j=0}^{k r-1} h_{j}{ }^{\prime} \Delta^{j} f(n r)=N^{-1} \sum_{n=1}^{N} \exp 2 \pi i \Delta^{p} f(n r) & \\
& =N^{-1} \sum_{n=1}^{N} \exp 2 \pi i p ! r^{p} \alpha=\exp 2 \pi i p ! r^{p} \alpha \neq 0 .
\end{aligned}
$$

Therefore, $w^{(n)}$ is not equidistributed if $k r>p$. But if $k r \leqq p$, and if $h_{j}{ }^{\prime}=0$ for $j<s$ but $h_{s}{ }^{\prime} \neq 0$, then $\sum h_{j}{ }^{\prime} \Delta^{j} f(n r)$ is a polynomial in $n$ of degree $p-s \geqq 1$ with leading coefficient

$$
\beta=p(p-1) \cdots(p-s+1) r^{p} \alpha h_{s}{ }^{\prime} .
$$

Since $\beta$ is irrational, we have the required zero limit (7.12). This completes the proof.

Theorem 22. For almost all $\theta>1$, for any $r>0$, the $r$-dimensional sequence $y^{(n)}$ derived from $x_{n}=\left\{\theta^{n}\right\}$ is completely equidistributed.

Proof. We must show that for almost all $\theta>1$ the sequence

$$
w^{(n)} \equiv\left(\theta^{n r}, \theta^{n r+1}, \cdots, \theta^{n r+k r-1}\right) \quad(\bmod 1)
$$

is equidistributed in $C_{k r}$. The Weyl criterion requires

$$
\lim _{N \rightarrow \infty} N^{-1} \sum_{n=1}^{N} \exp 2 \pi i \sum_{j=0}^{k r-1} h_{j} \theta^{n r+j}=0
$$

for any integers $h_{0}, \cdots, h_{k r-1}$ not all zero, i.e.,

$$
\lim _{N \rightarrow \infty} N^{-1} \sum_{n=1}^{N} \exp 2 \pi i p(\theta) \theta^{n r}=0
$$

where $p(\theta)=\sum h_{j} \theta^{j}=$ a polynomial which is not identically zero. But by Theorem 14 , if we set $M(n)=n r$ and $L=r$, the sequence $p(\theta) \theta^{{ }^{n r}} \quad(n=1,2, \cdots)$ is equidistributed modulo 1 for almost all $\theta>1$. The relation (7.14), therefore, follows from the Weyl criterion applied to the one-dimensional sequence $p(\theta) \theta^{n r}$.

California Institute of Technology

Pasadena, California

1. H. WeYL, "Über die Gleichverteilung von Zahlen modulo Eins," Math. Ann., v. 77, 1916, p. 313-352.

2. J. G. vaN DER Corput, "Diophantische Ungleichungen. I Zur Gleichverteilung modulo Eins," Acta Math., v. 56, 1931, p. 373-456.

3. J. F. Koksma, "Ein mengentheoretischer Satz über die Gleichverteilung modulo Eins," Compositio Math, v. 2, 1935, p. 250-258.

4. J. F. Koxsma, Diophantische Approximationen, Chelsea, New York, 1936.

5. D. L. JAGerman, The Autocorrelation Function of a Sequence Uniformly Distributed Modulo One, System Development Corp. Report SP-148, 1960, to appear in Annals of Mathematical Statistics.

6. O. TAUSSKY \& J. ToDD, “Generation of pseudo-random numbers," Symposium on Monte Carlo Methods, H. A. Meyer, Ed., John Wiley and Sons, New York, 1956, p. 15-28. 
7. J. N. Franklin, "On the equidistribution of pseudo-random numbers," Quar. Appl. Math., v. 16, 1958, p. 183-188.

8. A. Zygmund, Trigonometric Series, Volume II, Cambridge University Press, 1959.

9. G. Birkhoff \& S. MacLane, A Survey of Modern Algebra, Macmillan, 1948.

10. C. B. BELL, "On the structure of distribution-free statistics," Ann. Math. Statist., v. 31,1960 , p. 703-709.

11. W. F. BAUER, "The Monte Carlo method," J. Soc. Indus. Appl. Math., v. 6, no. 4, 1958.

12. G. E. P. Box \& M. E. Mueller, "A note on the generation of random normal deviates," Ann. Math. Statist., v. 29, 1958, p. 610-611.

13. G. W. Brown, "History of Rand's random digits-summary," Monte Carlo Method, Nat. Bur. Standards.; App. Math. Ser. 12, June 11, 1951.

14. Eve Bofinger \& V. I. Bofinger, "A periodic property of pseudo-random sequences," J. Assoc. Comput. Mach., v. 5, 1958, p. 261-265.

15. E. D. Cashwell \& C. J. Everetr, A Practical Manual on the Monte Carlo Method for Random Walk Problems, Pergamon Press, 1959.

16. J. E. Certaine, "On sequences of pseudo-random numbers of maximal lengths," $J$. Assoc. Comput. Mach., v. 5, 1958, p. 353.

17. C. E. Clark, "The utility of statistics of random numbers," Operations Res. v. 8, 1960 , p. 185-195.

18. R. R. Conveyou, "Serial correlation in the generation of pseudo-random numbers," J. Assoc. Comput. Mach., v. 7, 1960, p. 72-74.

19. A. P. Dempster, "Random allocation designs, I: On general classes of estimation methods," Ann. Math. Statist., v. 31, 1960, p. 885-905.

20. H. J. A. Dupark, C. G. Lekerkerker \& W. Peremans, "Reduced sequences of integers and pseudo-random numbers," Math. Centrum Amsterdam, Report ZW 1953, 002.

21. A. R. Edmonds, "The generation of pseudo-random numbers on electronic digital computers," Comput. J., v. 2, 1959/60, p. 181-185.

22. D. I. Golenko, "Calculation of the characteristics of certain stochastic processes by the Monte Carlo Method," Vyčisl. Mat., v. 5, 1959, p. 93-108.

23. I. J. Good, "The serial test for sampling numbers and other tests for randomness," Proc. Cambridge Philos. Soc., v. 49, 1953, p. 276-284.

24. B. F. Green, JR., J. E. K. Smith, \& LaUrA KLem, "Empirical tests of an additive random number generator," J. Assoc. Comput. Mach., v. 6, 1959, p. 527-537.

25. M. GREenberger, "An a priori determination of serial correlation in computer generated random numbers," Unpublished paper 1960 (MIT).

26. M. Greenberger, "Notes on a new pseudo-random number generator," J. Assoc. Comput. Mach., v. 8, no. 2, 1961, p. 163-167.

27. M. Greenberger, "Random number generators," preprints of the Fourteenth National Conference of the $A C M$, September 1959 .

28. P. C. Hammer, "The mid-square method of generating digits," Monte Carlo Method, National Bureau of Standards, Washington, D. C., 1951, p. 33.

29. D. G. N. Hunter, "Note on a test for repeating cycles in a pseudo-random number generator,' Comput. J., v. 3, 1960/61, p. 9.

30. M. IsIDA \& HiRoJI IKEDA, "Random number generator," Ann. Inst. Statist. Math., Tokyo, v. 8, 1956, p. 119-126.

31. D. L. Johnson, "Generating and testing pseudo-random numbers on the IBM type 701," Math. Tables Aids Comput., v. 10, 1956, p. 8-13.

32. M. L. JuncosA, Random Number Generation on the BRL High-Speed Computing Machines, Report No. 855, Ballistics Research Labs, Aberdeen Proving Ground, Maryland, 1953.

33. H. KaHN, Applications of Monte Carlo, Office of Technical Services, Dept. of Commerce, Washington 25 , D. C., revised 1956.

34. M. G. Kendall, The Advanced Theory of Statistics, Vol. II, Griffin and Company, London, 1948, Ch. 21 .

35. J. E. Kokıe, "One statement Fortran-type random number generators," Paper No. 59 contributed to Fifteenth ACM Nat. Conf., Milwaukee, Wis., August 1960.

36. H. G. KUEHN, "A 48-bit pseudo-random number generator," J. Assoc. Comput. Mach., v. 4 , no. 8,1961, p. $350-352$.

37. D. H. Lenmer, "Mathematical methods in large-scale computing units," Proceedings of Second Symp. on Large-Scale Digital Calculating Machinery, Ann. of the Computation Lab. of Harvard U., v. 26, 1951, p. 141.

38. G. MARSAGLIA, "Expressing random variables in terms of uniform random variables," Ann. Math. Statist., v. 32, no. 3, 1961, p. 894-898.

39. G. Marsaglia, "Generating exponential random variables," Ann. Math. Statist., v. 32 , no. 3 , 1961, p. 899-900.

40. J. Moshman, "The generation of pseudo-random numbers on a decimal calculator," J. Assoc. Comput. Mach., v. 1, 1954, p. 88-91.

41. M. E. Muller, Generation of Normal Deviates, TR 13, Statistical Techniques Res. Group, of Mathematics, Princeton University.

42. M. E. Muller, "A comparison of methods for generating normal deviates on digital computers," J. Assoc. Comput. Mach., v. 6, 1959, p. 376-383. 
43. M. E. MULLER, "An inverse method for the generation of random normal deviates on large scale computers," Math. Tables Aids Comput., v. 12, 1958, p. 167-174.

44. E. S. PAGE, "Pseudo-random elements for computers," Appl. Statist., v. 8, 1959, p. 124-131.

45. A. Rotenberg, "A new pseudo-random number generator," J. Assoc. Comput. Mach., v. 7, 1960, p. 75-77.

46. I. M. SoboL, "Pseudo-random numbers for the machine 'Strela'," teor. Veroyatnost. $i$ primenen, v. 3, (Russian-Eng. Summary), 1958, p. 205-211.

47. W. W. Stone, "Team: A three-dimensional Monte Carlo reactor program utilizing a fast random variable generation technique," paper No. 60 contributed to Fifteenth ACM Nat. Conf., Milwaukee, Wis., Aug. 1960.

48. J. ToDD, "Some experiments on Monte Carlo method," presented before the Inst. of Math. Statistics, Boston, 1951.

49. J. von Neuman, "Various techniques in connection with random digits," Monte Carlo Methods, Nat. Bur. of Standards, Appl. Math. Ser. v. 12, 1951, p. 36-38. 1959 .

50. N. Zierler, "Linear recursive sequences," J. Soc. Indust. Appl. Math., v. 7, no. 1, 